Research Article

\title{
Complexity Analysis of a Modified Predator-Prey System with Beddington-DeAngelis Functional Response and Allee-Like Effect on Predator
}

\author{
Shuangte Wang $(\mathbb{D}$ and Hengguo Yu \\ College of Mathematics and Physics, Wenzhou University, Wenzhou, China \\ Correspondence should be addressed to Shuangte Wang; wanshuangte@126.com
}

Received 25 June 2020; Revised 24 December 2020; Accepted 5 January 2021; Published 15 February 2021

Academic Editor: Rodica Luca

Copyright (c) 2021 Shuangte Wang and Hengguo Yu. This is an open access article distributed under the Creative Commons Attribution License, which permits unrestricted use, distribution, and reproduction in any medium, provided the original work is properly cited.

In this paper, complex dynamical behaviors of a predator-prey system with the Beddington-DeAngelis functional response and the Allee-like effect on predator were studied by qualitative analysis and numerical simulations. Theoretical derivations have given some sufficient and threshold conditions to guarantee the occurrence of transcritical, saddle-node, pitchfork, and nondegenerate Hopf bifurcations. Computer simulations have verified the feasibility and effectiveness of the theoretical results. In short, we hope that these works could provide a theoretical basis for future research of complexity in more predator-prey ecosystems.

\section{Introduction}

In reference [1], the authors simply considered a predatorprey system with Holling type II functional response and Allee-like effect on predator, which is described by the following nonlinear ordinary differential equations (ODEs):

$$
\begin{aligned}
& \dot{x}=r_{1} x\left(1-\frac{x}{K_{1}}\right)-\frac{q x y}{a+x}-m_{1} x-\mathrm{d} x^{2}, \\
& \dot{y}=r_{2} y\left(1-\frac{y}{K_{2}}\right) \frac{y}{y+e}+\frac{e_{1} q x y}{a+x}-m_{2} y,
\end{aligned}
$$

subject to initial conditions $x(0), y(0) \geq 0$. Here we replace the Holling type II functional response $(q x) /(a+x)$ with a functional response $(q x) /(a+b x+c y)$ and denote the parameter $q$ as $q_{1}$ for later use, and thus above system (1a) and system (1b) have a modified version:

$$
\begin{aligned}
& \dot{x}=r_{1} x\left(1-\frac{x}{K_{1}}\right)-\frac{q_{1} x y}{a+b x+c y}-m_{1} x-\mathrm{d} x^{2}, \\
& \dot{y}=r_{2} y\left(1-\frac{y}{K_{2}}\right) \frac{y}{y+e}+\frac{e_{1} q_{1} x y}{a+b x+c y}-m_{2} y,
\end{aligned}
$$

where functions $x=x(t)$ and $y=y(t)$ are the densities of prey and predator at time $t$, respectively. In terms of biology, all above positive constants have practical considerations. Parameters $r_{1}$ and $r_{2}$ denote the intrinsic growth rate of the prey and predator, respectively; $K_{1}$ and $K_{2}$ represent the carrying capacity of the environment; $a$ is the half-saturation constant; $q_{1}$ is the search efficiency of predator for prey; $m_{1}$ and $m_{2}$ are the mortality rate of the prey and predator species, respectively; $e_{1}$ is the biomass conversion and we denote $e_{1} q_{1}$ as $q_{2}$ for convenience; $d$ is the intraspecific competition coefficient of the prey; $e$ is the Allee effect 
constant. The specific growth term $r_{1} x\left(1-\left(x / K_{1}\right)\right)$ governs the increase of prey in the lack of predator, while the specific growth term $r_{2} y\left(1-\left(y / K_{2}\right)\right)$ governs the increase of predator. The square term $\mathrm{d} x^{2}$ is an intrinsic decrease term on prey. The term $y /(y+e)$ on the specific growth term of predator with multiply form is called the Allee-like effect $[2,3]$ and is different from predator-prey systems in [4-8] with Allee effect on prey. The coupled term $q_{1} x /(a+b x+c y)$ is named Beddington-DeAngelis (B-D) functional response (named after Beddington and DeAngelis et al.) [9, 10]. It is similar to the Holling type II functional response incorporating an extra term $c y$ in denominator, which describes mutual interference among predators $[11,12]$. This functional response has some of the same qualitative features as the ratio-dependent form but avoids some of singular behaviors of ratio-dependent models at low densities [11]. Obviously, when $b=1$ and $c=0$, system (2a) and system (2b) reduce to original system (1a) and system (1b).

When parameters $d=m_{1}=e=0$ (without Allee effect) and $r_{2}-m_{2}<0$, system (2a) and system (2b) reduce to System (2.1) in [13] and the author particularly conducted stability (local and global) and bifurcation (saddle-node, transcritical, Hopf-Andronov, and Bogdanov-Takens) analysis with a detailed mathematical analysis. When $d=m_{1}=r_{2}=0$, system (2a) and system (2b) become model system (3a) and model system (3b) in [14], which was also independently and originally proposed in [9-11], while in [14], the authors discussed local and global asymptotic stability behavior of various equilibria and Hopf bifurcation occurs when parameter $m$ corresponding to reserved region crosses some critical values. To mimic the real-world scenario, they solved the inverse problem of estimation of system parameter $m$ by using the sampled data. System (1.3) in [15] is similar to above system in [14] except the constant rate harvesting term. In this reference, the authors showed that it undergoes several kinds of bifurcations, such as the saddle-node bifurcation, the subcritical and supercritical Hopf bifurcation, and Bogdanov-Takens bifurcation by choosing the death rate of the predator and the harvesting rate of the prey as bifurcation parameters.

Motivated by previous progress of predator-prey systems with B-D functional response or Allee-like effect, this paper mainly concentrates on dynamical analysis of system (2a) and system (2b). The rest of this paper is structured as follows. Preliminaries, such as boundedness, permanence, and existence of trivial equilibria, are given in Section 2. The existence of interior equilibrium is presented in Section 3 by virtues of the cobweb model and polynomial equations, respectively. In Section 4, we give stability analysis of equilibria and nonexistence of limit cycles. In Section 5, local codimension one bifurcations are analyzed, especially the Hopf bifurcation incorporating numerical simulations and Hopf bifurcation curves. In Section 6, we carry out short conclusions for our system.

\section{Preliminaries}

In this section, we devote to give some priori foundations. Before presenting the main results, we denote the first quadrant as $\mathbb{R}^{+2}$, and its closure is $\mathbb{R}_{+}^{2}=\overline{\mathbb{R}^{+2}}$. For biological consideration, system (2a) and system (2b) are defined on the domain $\mathbb{R}_{+}^{2}$ and all the solutions are non-negative with initial conditions $x(0), y(0) \geq 0$, i.e., $\mathbb{R}_{+}^{2}$ is an invariant set and any orbits starting from it cannot cross the coordinate axes. Furthermore, all solutions are uniformly bounded. But now we only need to prove following theorems.

\subsection{Boundedness and Permanence}

Theorem 1 (uniform boundedness). Suppose that a nonnegative function $\varphi(x, y)$ and its partial derivatives $\varphi_{x}$ and $\varphi_{y}$ are continuous in $\mathbb{R}_{+}^{2}$, then the system

$$
\begin{aligned}
& \dot{x}=r_{1} x\left(1-\frac{x}{K_{1}}\right)-\varphi(x, y)-m_{1} x-\mathrm{d} x^{2}, \\
& \dot{y}=r_{2} y\left(1-\frac{y}{K_{2}}\right) \frac{y}{y+e}+e_{1} \varphi(x, y)-m_{2} y,
\end{aligned}
$$

subject to $x(0), y(0) \geq 0$ is uniformly bounded.

This Theorem 1 holds obviously after introducing an auxiliary function $z=e_{1} x+y[1,16]$. The following theorem with the help of comparison principle in ODEs is about permanence of system (2a) and system (2b).

Theorem 2 (permanence). If parameters satisfy

$$
\begin{aligned}
& \omega_{1}=\frac{r_{1}-m_{1}-\left(\left(q_{1} M_{2}\right) /\left(a+c M_{2}\right)\right)}{\left(r_{1} / K_{1}\right)+d}>0, \\
& a_{0}=\frac{q_{2} \omega_{1}(1-\lambda)}{a+b \omega_{1}(1-\lambda)}-m_{2}>0, \\
& a_{1}=\frac{r_{2}}{e}-\frac{c q_{2} \omega_{1}(1-\lambda)}{\left[a+b \omega_{1}(1-\lambda)\right]^{2}}<0, \\
& c \rho \leq 1, e \geq \rho\left[a+b \omega_{1}(1-\lambda)\right],
\end{aligned}
$$


where $M_{2}$ is a positive constant, $\lambda \in(0,1)$, and $\rho=\sqrt[3]{\left(\left(r_{2} e\left(K_{2}+e\right)\right) /\left(K_{2} c^{2} q_{2} \omega_{1}(1-\lambda)\right)\right)}$, then system (2a) and system (2b) are permanent.

Proof. From above Theorem 1, we have a positive upper bound $\xi_{1}$ such that

$$
\max \left\{\limsup _{t \rightarrow \infty} x, \limsup _{t \rightarrow \infty} y\right\} \leq \xi_{1} .
$$

Then, we obtain $M_{2}>0$ and sufficiently large $T_{1}$, such that

$$
\dot{x} \geq x\left[r_{1}-m_{1}-\frac{q_{1} M_{2}}{a+c M_{2}}-\left(\frac{r_{1}}{K_{1}}+d\right) x\right], \quad \forall t \geq T_{1} .
$$

By using the lemmas in [17], we admit $\liminf _{t \longrightarrow \infty} x \geq \omega_{1}$. That is to say, there exist sufficiently large $T_{2}$, such that $x \geq \omega_{1}(1-\lambda), \forall t \geq T_{2}$. Then, we consider equation (2b) again. It yields $\dot{y} \geq f(y) y$; here the function $f(y)$ incorporating its Taylor expression is

$$
\begin{aligned}
f(y) & =\frac{r_{2} y}{y+e}\left(1-\frac{y}{K_{2}}\right)-m_{2}+\frac{q_{2} \omega_{1}(1-\lambda)}{a+b \omega_{1}(1-\lambda)+c y} \\
& =a_{0}+a_{1} y+\frac{1}{2} f^{\prime \prime}\left(\xi_{y}\right) y^{2}, \quad \xi_{y} \in[0, y],
\end{aligned}
$$

in which the second order derivative of $f(y)$ is

$$
f^{\prime \prime}(y)=-\frac{2 r_{2} e\left(K_{2}+e\right)}{K_{2}(y+e)^{3}}+\frac{2 c^{2} q_{2} \omega_{1}(1-\lambda)}{\left[a+b \omega_{1}(1-\lambda)+c y\right]^{3}} .
$$

From the conditions in this theorem, we know that $f^{\prime \prime}(y) \geq 0$, when $y \geq 0$. Thus, $f(y) \geq a_{0}+a_{1} y, \forall y \geq 0$. By using the lemmas in [17] again, the proof is completed.

2.2. Existence of Trivial Equilibria. In this section, we will discuss the existence conditions of trivial equilibria of system (2a) and system (2b). Firstly, from [1], it is obvious that system (2a) and system (2b) have trivial equilibria: $E_{0}:=(0,0), E_{1}:=\left(x_{1}, 0\right)$, and $E_{2}^{(k)}:=\left(0, y_{k}\right), k=1,2$, where $x_{1}:=\left(\left(r_{1}-m_{1}\right) /\left(\left(r_{1} / K_{1}\right)+d\right)\right)$ and

$$
y_{1,2}:=\frac{r_{2}-m_{2} \pm \sqrt{\Delta}}{\left(2 r_{2} / K_{2}\right), \Delta:=\left(r_{2}-m_{2}\right)^{2}-\frac{4 r_{2} m_{2} e}{K_{2}},}
$$

The point $E_{1}$ exists when $r_{1}>m_{1}$, while the points $E_{2}^{(k)}$ all exist if $r_{2}>m_{2}$ and $\Delta>0$. If $r_{2}>m_{2}$ but $\Delta=0$, then the two equilibria $E_{2}^{(1)}$ and $E_{2}^{(2)}$ collide with each other and we denote this equilibrium as $E_{2}=\left(0, y_{2}\right)$.

\section{Existence of Interior Equilibrium}

Here and below, we denote the interior equilibrium $E_{*}$ of system (2a) and system (2b) as $\left(x_{*}, y_{*}\right)$ or $\left(s_{1}, s_{2}\right)$. This equilibrium must satisfy the following coupled algebraic equations:

$$
\begin{aligned}
& r_{1}\left(1-\frac{x}{K_{1}}\right)-\frac{q_{1} y}{a+b x+c y}-m_{1}-\mathrm{d} x=0, \\
& r_{2}\left(1-\frac{y}{K_{2}}\right) \frac{y}{y+e}+\frac{q_{2} y}{a+b x+c y}-m_{2}=0 .
\end{aligned}
$$

From equation (10a), we have (1) $y \longrightarrow\left(\left(\left(r_{1}-m_{1}\right) a\right) /\left(q_{1}-\left(r_{1}-m_{1}\right) c\right)\right) \quad$ or $\quad \infty \quad$ (if $\left.q_{1}=\left(r_{1}-m_{1}\right) c\right)$ when $x \longrightarrow 0$; (2) $x \longrightarrow x_{1}$ when $y \longrightarrow 0$. Bearing equation (10b) in mind, we have (1) $y \longrightarrow y_{k}$ (if $y_{k}$ exists) when $x \longrightarrow 0$; (2) $x \longrightarrow\left(\left(a m_{2}\right) /\left(q_{2}-m_{2} b\right)\right)$ or $\infty$ (if $\left.q_{2}=m_{2} b\right)$. The implicit derivative $y^{\prime}(x)$ from equation (10b) is

$$
y^{\prime}(x)=\frac{q_{2} K_{2}(a+c y)(y+e)^{2}}{r_{2}\left(y^{2}+2 e y-K_{2} e\right)(a+b x+c y)^{2}+q_{2} c K_{2} x(y+e)^{2}},
$$

and we denote the positive root of a quadratic equation $y^{2}+$ $2 e y-K_{2} e=0$ as $y_{s_{1}}=-e+\sqrt{e^{2}+K_{2} e}$ for later use.

3.1. Cobweb Model. Based on above approximate analysis and the cobweb model, some cases about the existence of the interior equilibrium $E_{*}$ will be illustrated when isoclines from equations (10a) and (10b) all fall in $R_{+}^{2}$.

Case 1. If parameters satisfy

$$
\frac{\left(r_{1}-m_{1}\right) a}{q_{1}-c\left(r_{1}-m_{1}\right)}>y_{k}>y_{s_{1}}, \quad x_{1}>0,
$$

then an interior equilibrium exists. Here, we take $r_{1}=1$, $r_{2}=1, K_{1}=20, K_{2}=8, q_{1}=0.12, q_{2}=0.06, a=4$, $m_{1}=0.3, m_{2}=0.5, e=0.3, b=1, c=0.1$, and $d=5$; the first equilibrium is $E_{3} \approx(0.119085,3.688431)$ and the second equilibrium is $E_{*} \approx(0.136769,0.323748)$.

Case 2. If parameters satisfy

$$
q_{1}=c\left(r_{1}-m_{1}\right), \quad y_{k}>y_{s_{1}}, x_{1}>0,
$$

then an interior equilibrium exists. Here, we take $r_{1}=1$, $r_{2}=1, K_{1}=20, K_{2}=8, q_{2}=0.6, a=4, m_{1}=0.3$, $m_{2}=0.5, e=0.3, b=1, c=0.1$, and $d=5$; the first interior equilibrium is $E_{4} \approx(0.126835,3.83244)$, and the second equilibrium is $E_{7} \approx(0.137621,0.298566)$.

Case 3. If parameters satisfy

$$
\begin{aligned}
& 0<x_{1}<\frac{a m_{2}}{q_{2}-m_{2} b}, \\
& 0<y_{k}<\min \left\{y_{s_{1}}, \frac{\left(r_{1}-m_{1}\right) a}{q_{1}-c\left(r_{1}-m_{1}\right)}\right\},
\end{aligned}
$$

then an interior equilibrium exists. Here, we take $r_{1}=1, r_{2}=1.5, K_{1}=20, K_{2}=50, q_{1}=5, q_{2}=1.1$, $a=4, b=1, c=0.1, m_{1}=0.1, m_{2}=0.5, e=1$, and $d=3.565 ;$ an interior equilibrium is $E_{5} \approx(0.0916118,0.470837)$.

Case 4. If parameters satisfy 


$$
\begin{aligned}
& 0<x_{1}, \\
& q_{2}=m_{2} b, \\
& 0<y_{k}<\min \left\{y_{s_{1}}, \frac{\left(r_{1}-m_{1}\right) a}{q_{1}-c\left(r_{1}-m_{1}\right)}\right\},
\end{aligned}
$$

then an interior equilibrium exists. Here, we take $r_{1}=1, \quad r_{2}=1, \quad K_{1}=20, \quad K_{2}=8, \quad q_{1}=0.12, \quad a=4$, $m_{1}=0.3, m_{2}=0.5, e=0.3, b=1, c=0.1$, and $d=5$; an interior equilibrium is $E_{6} \approx(0.136885,0.303164)$. Notice that another interior equilibrium $E_{*} \approx(0.118552,3.79777)$ verifies Case 1 .

Case 5. If parameters satisfy

$$
\begin{aligned}
& 0<x_{1}<\frac{a m_{2}}{q_{2}-m_{2} b}, \\
& 0<y_{k}<y_{s_{1}}, \\
& q_{1}=c\left(r_{1}-m_{1}\right),
\end{aligned}
$$

then an interior equilibrium exists (see the second equilibrium $E_{7}$ in Case 2).

Case 6. If $y_{k}$ does not exist and parameters satisfy

$$
\begin{gathered}
0<\frac{a m_{2}}{q_{2}-m_{2} b}<x_{1}, \\
q_{1}>c\left(r_{1}-m_{1}\right),
\end{gathered}
$$

then an interior equilibrium exists. Here, we take $r_{1}=1, r_{2}=1.5, K_{1}=10, K_{2}=2, q_{1}=5, q_{2}=1.1$, $a=4, b=1, c=0.1, m_{1}=0.1, m_{2}=0.5, e=1$, and $d=0.05389805885 \approx d_{8}^{[H]}$; an interior equilibrium is $E_{8} \approx(0.399541,0.750397)$.

Case 7. If $y_{k}$ does not exist and parameters satisfy

$$
0<\frac{a m_{2}}{q_{2}-m_{2} b}<x_{1}, \quad q_{1}=c\left(r_{1}-m_{1}\right),
$$

then an interior equilibrium exists. Here, we take $r_{1}=1, r_{2}=1.5, K_{1}=10, K_{2}=2, q_{2}=1.1, a=4, b=1$, $c=0.1, m_{1}=0.1, m_{2}=0.5, e=1$, and $d=0.11$; an interior equilibrium is $E_{9} \approx(4.178700,2.09452)$.

3.2. Polynomial Equations of $x_{*}$ and $y_{*}$. From equation (10a), an expression of $y$ is

$$
y=\frac{\left(x_{1}-x\right)\left(\left(r_{1} / K_{1}\right)+d\right)(a+b x)}{q_{1}-c\left(r_{1}-m_{1}\right)+c\left(\left(r_{1} / K_{1}\right)+d\right) x .}
$$

Substituting it into the equation (10b), a quintic algebraic equation of $x_{*}$ can be derived in the form of $p(x):=\sum_{k=0}^{5} a_{k} x^{k}=0$ (see coefficients $a_{k}$ in Appendix A.1). Thanks to Niels Henrik Abel and Evariste Gallois's ingenious works, quintic equations usually have no analytical form solutions. But we can make some special efforts to numerically derive positive roots for this equation $p(x)=0$. For instance, if

$$
p\left(x_{1}\right)=\frac{K_{2} e q_{1}^{3} K_{1}^{3}}{K_{1} d+r_{1}}\left[a m_{2}\left(K_{1} d+r_{1}\right)+K_{1}\left(r_{1}-m_{1}\right)\left(b m_{2}-q_{2}\right)\right]>0 \text {, }
$$

where $x_{1}>0$ and $a_{0}<0$, then there is a positive root $x_{*}$ such that $x_{*}<x_{1}$.

Case 1. If parameters satisfy

$$
\begin{aligned}
& p\left(x_{1}\right)>0, \\
& x_{1}>0, \\
& a_{0}<0, \\
& q_{1} \geq c\left(r_{1}-m_{1}\right),
\end{aligned}
$$

then an interior equilibrium exists. Here, we take $r_{1}=1, r_{2}=1, K_{1}=10, K_{2}=8, m_{1}=0.3, m_{2}=0.5$, $d=0.1, q_{1}=0.12, q_{2}=0.16, e=0.3, a=0.2, b=0.3$, and $c=0.1$; an interior equilibrium is $E_{10} \approx(3.491956,0.016748)$, and the following lemma is verified.

Lemma 1. Suppose that $f(x)=\sum_{k=0}^{n} a_{k} x^{k}$ is a polynomial with real coefficients, $a_{n} \neq 0, n>1$. If $a_{n} a_{0}<0$, then the equation $f(x)=0$ has a positive root.

Proof

We only consider the special case $a_{n}>0$, and thus $a_{0}<0$. It is clear that the polynomial $f(x)$ has a decomposition

$$
f(x)=\sum_{k=0}^{n-1}\left(\frac{1}{n} a_{n} x^{n-k}+a_{k}\right) x^{k} .
$$

If we take a sufficiently large positive number $X$ such that $X>\max _{0 \leq k \leq n-1}\left\{\left(n\left|a_{k}\right| / a_{n}\right)^{1 /(n-k)}\right\}$, then $f(X)>0$, and thus we complete the proof.

Lemma 2. Suppose that $f(x)=\sum_{k=0}^{n} a_{k} x^{k}$ is a real polynomial, $a_{n} \neq 0, n>1$. If there is a positive number $x_{0}>0$ such that $a_{n} f\left(x_{0}\right)<0$, then the equation $f(x)=0$ has a positive root.

On the other hand, from equation (10b), we obtain an expression

$$
x=\frac{(a+c y)\left[m_{2}(y+e)-r_{2} y\left(1-\left(y / K_{2}\right)\right)\right]}{\left(q_{2}-b m_{2}\right)(y+e)+b r_{2} y\left(1-\left(y / K_{2}\right)\right)} .
$$

And a quintic algebraic equation of $y_{*}$ can be written in the form of $q(y):=\sum_{k=0}^{5} b_{k} y^{k}=0$ (see coefficients $b_{k}$ in Appendix A.2). Suppose that the denominator and numerator of expression (23) are all positive for some $y_{*}>0$; then, $x_{*}>0$. Notice that if $b_{5}>0$ and $b_{0}<0$, there must exist a positive root for the quintic equation $q(y)=0$ (see Lemma 1$)$.

Case 2. If parameters satisfy $b_{0}<0, m_{2} \geq r_{2}, q_{2}>b m_{2}$, $y_{u_{1}}>0$, and $q\left(y_{u_{1}}\right)>0$, where 


$$
y_{u_{1}}=\frac{q_{2}-b m_{2}+b r_{2}+\sqrt{\left(q_{2}-b m_{2}+b r_{2}\right)^{2}+\left(4 b r_{2} e / K_{2}\right)\left(q_{2}-b m_{2}\right)}}{\left(2 b r_{2} / K_{2}\right)},
$$

is a root of the quadric equation in the denominator of (23), then an interior equilibrium exists. Here, we take $r_{1}=1, r_{2}=0.5, K_{1}=10, K_{2}=8, m_{1}=0.3, m_{2}=0.6$, $d=0.1, q_{1}=0.8, q_{2}=0.6, e=0.3, a=0.2, b=0.2$, and $c=0.1$, and an interior equilibrium is $E_{11} \approx(0.169319,0.212419)$.

Case 3. If parameters satisfy $b_{0}<0, y_{2}>0, q\left(y_{2}\right)>0$, $y_{u_{1}}>0$, and $q\left(y_{u_{1}}\right)>0$, then an interior equilibrium exists. Here, we take $r_{1}=1, r_{2}=1, K_{1}=20, K_{2}=80$, $q_{1}=3, q_{2}=10, a=2, b=1, c=1, m_{1}=0.1, m_{2}=0.5$, $e=2$, and $d=2.092753894 \approx d_{12}^{[H]}$, and an interior equilibrium is $E_{12} \approx(0.067460,0.695847)$.

Remark 1. Here we rewrite equations (10a) and (10b) as

$$
\begin{aligned}
f(x, y)= & {\left[r_{1}\left(1-\frac{x}{K_{1}}\right)-m_{1}-d x\right](a+b x+c y)-q_{1} y=0 } \\
g(x, y)= & r_{2} y\left(1-\frac{y}{K_{2}}\right)(a+b x+c y)+q_{2} x(y+e) \\
& -m_{2}(a+b x+c y)(y+e)=0 .
\end{aligned}
$$

Furthermore, if we sort them as $f(x, y)=a_{0}(x) y+a_{1}(x)$, $g(x, y)=b_{0}(x) y^{3}+b_{1}(x) y^{2}+b_{2}(x) y+b_{3}(x)$, the first eliminant

$$
R_{y}(f, g)=\left|\begin{array}{cccc}
a_{0}(x) & a_{1}(x) & 0 & 0 \\
0 & a_{0}(x) & a_{1}(x) & 0 \\
0 & 0 & a_{0}(x) & a_{1}(x) \\
b_{0}(x) & b_{1}(x) & b_{2}(x) & b_{3}(x)
\end{array}\right|,
$$

also yields above quintic equation $p(x)=0$. Similarly, if we sort them as $f(x, y)=a_{0}(y) x^{2}+a_{1}(y) x+a_{2}(y)$, $g(x, y)=b_{0}(y) x+b_{1}(y)$, the second eliminant

$$
R_{x}(f, g)=\left|\begin{array}{ccc}
a_{0}(y) & a_{1}(y) & a_{2}(y) \\
b_{0}(y) & b_{1}(y) & 0 \\
0 & b_{0}(y) & b_{1}(y)
\end{array}\right|,
$$

yields the quintic equation $q(y)=0$.

\section{Stability Analysis of System (2a) and System (2b)}

In this section, we use the Routh-Hurwitz criterion and Perron's theorems to analyze local stability of above equilibria in their existence interval, respectively. A theorem about global asymptotic stability and a theorem about nonexistence of limit cycles are also considered.
4.1. Local Stability Analysis. The Jacobian matrix of system (2a) and system (2b) takes the following form $J=\left(J_{i j}\right)_{2 \times 2}$, where four components are

$$
\begin{aligned}
J_{11}= & r_{1}-m_{1}-2 x\left(\frac{r_{1}}{K_{1}}+d\right)-\frac{q_{1}(a+c y) y}{(a+b x+c y)^{2}}, \\
J_{12}= & -\frac{q_{1} x(a+b x)}{(a+b x+c y)^{2}}, \\
J_{21}= & \frac{q_{2} y(a+c y)}{(a+b x+c y)^{2}}, \\
J_{22}= & \frac{r_{2} y}{K_{2}(y+e)^{2}}\left(-2 y^{2}-3 e y+K_{2} y+2 K_{2} e\right) \\
& +\frac{q_{2} x(a+b x)}{(a+b x+c y)^{2}}-m_{2} .
\end{aligned}
$$

For the trivial equilibrium $E_{0}$ with $r_{1} \neq m_{1}$ and the axial equilibrium $E_{1}$ with $\left(q_{2} x_{1} /\left(b x_{1}+a\right)\right) \neq m_{2}$, we omit their stability [1]. When $r_{1}=m_{1}$, the transformation $\tau=-m_{2} t$ and equations (2a) and (2b) yield $\dot{x}=\left(\left(\left(r_{1} / K_{1}\right)+d\right) / m_{2}\right) x^{2}$ (we still use symbol $t$ ), and thus $E_{0}$ is a saddle node and the parabolic sector is on the right half plane.

In the case that two axial equilibria $E_{2}^{(k)}(k=1,2)$ exist, the Jacobian matrices are

$$
J\left(E_{2}^{(k)}\right)=\left[\begin{array}{cc}
r_{1}-m_{1}-\frac{q_{1} y_{k}}{a+c y_{k}} & 0 \\
\frac{q_{2} y_{k}}{a+c y_{k}} & \frac{\left(m_{2}-r_{2}\right) y_{k}+2 m_{2} e}{y_{k}+e}
\end{array}\right] .
$$

Since $J_{22}\left(E_{2}^{(1)}\right)<0$, we have the following: (a) $E_{2}^{(1)}$ is a saddle point if $r_{1}-m_{1}>\left(\left(q_{1} y_{1}\right) /\left(a+c y_{1}\right)\right)$; (b) $E_{2}^{(1)}$ is an asymptotically stable node if $r_{1}-m_{1}<\left(\left(q_{1} y_{1}\right) /\left(a+c y_{1}\right)\right)$. For the equilibrium $E_{2}^{(2)}$, it is obvious that $J_{22}\left(E_{2}^{(2)}\right)>0$, and we have the following: (a) $E_{2}^{(2)}$ is an unstable node if $r_{1}-m_{1}>\left(\left(q_{1} y_{2}\right) /\left(a+c y_{2}\right)\right)$; (b) $E_{2}^{(2)}$ is a saddle point if $r_{1}-m_{1}<\left(\left(q_{1} y_{2}\right) /\left(a+c y_{2}\right)\right)$; (c) $E_{2}^{(2)}$ is an unstable higherorder singular point if $r_{1}-m_{1}=\left(\left(q_{1} y_{2}\right) /\left(a+c y_{2}\right)\right)$. In the special case that two axial equilibria $E_{2}^{(k)}(k=1,2)$ collide with each other, the Jacobian matrix is

$$
J\left(E_{2}\right)=\left[\begin{array}{cc}
r_{1}-m_{1}-\frac{q_{1} y_{2}}{a+c y_{2}} & 0 \\
\frac{q_{2} y_{2}}{a+c y_{2}} & 0
\end{array}\right],
$$

and thus $E_{2}$ is a higher-order singular point. If $r_{1}-m_{1}>\left(\left(q_{1} y_{2}\right) /\left(a+c y_{2}\right)\right), E_{2}$ is unstable. 
For the interior equilibrium $E_{*}$, its Jacobian matrix is

$$
J\left(E_{*}\right)=\left[\begin{array}{cc}
J_{11}\left(E_{*}\right) & \frac{-q_{1} x_{*}\left(a+b x_{*}\right)}{\left(a+b x_{*}+c y_{*}\right)^{2}} \\
\frac{q_{2} y_{*}\left(a+c y_{*}\right)}{\left(a+b x_{*}+c y_{*}\right)^{2}} & J_{22}\left(E_{*}\right)
\end{array}\right],
$$

where

$$
\begin{aligned}
& J_{11}\left(E_{*}\right)=-\left(\frac{r_{1}}{K_{1}}+d\right) x_{*}+\frac{q_{1} b x_{*} y_{*}}{\left(a+b x_{*}+c y_{*}\right)^{2}}, \\
& J_{22}\left(E_{*}\right)=\frac{r_{2} y_{*}}{K_{2}\left(y_{*}+e\right)^{2}}\left(-y_{*}^{2}-2 e y_{*}+K_{2} e\right)-\frac{q_{2} c x_{*} y_{*}}{\left(a+b x_{*}+c y_{*}\right)^{2}} .
\end{aligned}
$$

Denoting a new discriminant $\Delta_{*}:=A_{1}^{2}-4 A_{2}$ with the $\operatorname{trace} A_{1}:=\operatorname{tr} J\left(E_{*}\right)$ and the determinant $A_{2}:=\operatorname{det} J\left(E_{*}\right)$, we have

(a) If $A_{1}<0$ and (a1) $A_{2}>0, \Delta_{*} \geq 0$, then $E_{*}$ is an asymptotically stable node; (a2) $A_{2}>0, \Delta_{*}<0$, then $E_{*}$ is an asymptotically stable focus; (a3) $A_{2}<0$, then $E_{*}$ is a saddle point.

(b) If $A_{1}=0$ and (b1) $A_{2}>0$, then $E_{*}$ is a center or a focus; (b2) $A_{2}<0$, then $E_{*}$ is a saddle point.

(c) If $A_{1}>0$, then $E_{*}$ is unstable and (c1) $\Delta_{*}=0$, then $E_{*}$ is a node; (c2) $\Delta_{*}<0$, then $E_{*}$ is a focus; (c3) $\Delta_{*}>0$ and $A_{2}>0$, then $E_{*}$ is a node; (c4) $\Delta_{*}>0$ and $A_{2}<0$, then $E_{*}$ is a saddle point.

When $A_{2}=0$ but $A_{1} \neq 0, E_{*}$ is a stable (unstable) node if $A_{1}<0\left(A_{1}>0\right)$ (see Theorem 7.1 in Zhifen Zhang's book [18] for more details). It is probable that $E_{*}$ has a cusp case of codimension at least 2 which ensures potential Bogdanov-Takens bifurcation when $A_{1}=A_{2}=0$.

4.2. Global Asymptotic Stability. Combining the stability conclusions of the point $E_{0}$ in above section, the positive definite Lyapunov function $V=e_{1} x+y$ ensures that $E_{0}$ is globally asymptotically stable if one of the following conditions holds:

$$
\begin{aligned}
& \text { (a) } r_{1} \leq m_{1}, r_{2} \leq m_{2} \\
& \text { (b) } r_{1} \leq m_{1}, \Delta<0
\end{aligned}
$$

Furthermore, conditions $r_{1}<m_{1}, r_{2}+\left(q_{2} / b\right)-m_{2} \leq 0$ and Theorem 1 deduce global asymptotical stability of $E_{0}$ pronto. If $r_{1}>m_{1}, r_{2}+\left(q_{2} / b\right)-m_{2} \leq 0$, and $\left(\left(q_{2} x_{1}\right) /\left(a+b x_{1}\right)\right)-m_{1} \leq 0$, equilibria $E_{2}^{(k)}, E_{2}$, and $E_{*}$ do not exist, and $E_{0}$ is unstable, then
Theorem 1 ensures that $E_{1}$ is globally asymptotically stable. For the further consideration of point $E_{*}$, the following theorem explains its global asymptotic stability.

Theorem 3 (global asymptotic stability of $E_{*}$ ). If a unique interior equilibrium $E_{*}$ exists and parameters satisfy

$$
\frac{r_{1}}{K_{1}}+d>\frac{q_{1} b y_{*}}{a\left(a+b x_{*}+c y_{*}\right)}, \quad y_{*}>K_{2},
$$

then $E_{*}$ is globally asymptotically stable.

Proof. Here we take an unbounded positive definite Lyapunov function

$$
\begin{aligned}
V= & V(x, y)=\left(x-x_{*}-x_{*} \ln \frac{x}{x_{*}}\right) \\
& +A\left(y-y_{*}-y_{*} \ln \frac{y}{y_{*}}\right),
\end{aligned}
$$

with $A=\left(\left(q_{1}\left(a+b x_{*}\right)\right) /\left(q_{2}\left(a+c y_{*}\right)\right)\right)$. Introducing new variables $\hat{x}=x-x_{*}$ and $\hat{y}=y-y_{*}$, computing derivative along orbits of system (2a) and system (2b), we have

$$
\begin{aligned}
\left.\frac{\mathrm{d} V}{\mathrm{~d} t}\right|_{(2)}= & {\left[-\frac{r_{1}}{K_{1}}-d+\frac{q_{1} b y_{*}}{(a+b x+c y)\left(a+b x_{*}+c y_{*}\right)}\right] \hat{x}^{2} } \\
& +A\left\{\frac{r_{2}}{(y+e)\left(y_{*}+e\right)}\left[e-\frac{y y_{*}+e\left(y+y_{*}\right)}{K_{2}}\right]\right. \\
& \left.-\frac{q_{2} c x_{*}}{(a+b x+c y)\left(a+b x_{*}+c y_{*}\right)}\right\} \hat{y}^{2} .
\end{aligned}
$$

It is obvious that $\left.(\mathrm{d} V / \mathrm{d} t)\right|_{(2)}$ is negative definite. Consequently, the Lyapunov function $V$ satisfies the asymptotic stability theorem in [19]. Thus, we complete the proof.

If conditions $\left(\left(q_{1} b y_{*}\right) /\left(a\left(a+b x_{*}+c y_{*}\right)\right)\right) \leq\left(r_{1} / K_{1}\right)+$ $d$ and $y_{*} \geq K_{2}$ hold, then $A_{1}<0, A_{2}>0$ and $E_{*}$ is an asymptotically stable node or focus. Additionally, we could solve a potential interior equilibrium $E_{*}$ with two control variables $\lambda \in(0,1]$ and $\mu \geq 1$ from such conditions, where

$$
x_{*}=\frac{-a\left(K_{1} d+r_{1}\right)\left(K_{2} c \mu+a\right) \lambda+q_{1} b \mu K_{2} K_{1}}{a b \lambda\left(K_{1} d+r_{1}\right)}, \quad y_{*}=\mu K_{2} \text {, }
$$

and parameters $m_{1}$ and $m_{2}$ are constrained by 


$$
\begin{aligned}
& m_{1}=\frac{-a^{2}\left(K_{1} d+r_{1}\right) \lambda^{2}+a\left[\left(K_{1} d+r_{1}\right) a+\left(K_{2} c d \mu+b r_{1}\right) K_{1}+c K_{2} \mu r_{1}\right] \lambda-q_{1} b \mu K_{2} K_{1}}{a b \lambda K_{1}}, \\
& m_{2}=\frac{1}{\mu K_{2} K_{1} q_{1} b^{2}\left(K_{2} \mu+e\right)} \cdot \sum_{k=0}^{3}
\end{aligned}
$$

where coefficients are

$$
\begin{aligned}
& a_{3}=-K_{1} K_{2}^{2} b^{2} q_{1} r_{2}, \\
& a_{2}=-\left[\left(a c d \lambda-b q_{1}\right) q_{2}-r_{2} b^{2} q_{1}\right] K_{1}+a \lambda c q_{2} r_{1} K_{2}^{2}, \\
& a_{1}=-\left[a\left(K_{1} d+r_{1}\right)(c e+a) \lambda-b e K_{1} q_{1}\right] K_{2} q_{2}, \\
& a_{0}=-a^{2} q_{2} \lambda e\left(K_{1} d+r_{1}\right) .
\end{aligned}
$$

Here we set some values of parameters as $r_{1}=1.21$, $r_{2}=1.5, K_{1}=2, K_{2}=5, a=1, b=1.5, c=1.7, d=0.3$, $e=0.3, q_{1}=1.2$, and $q_{2}=0.21$, and the unique globally asymptotically stable node $E_{*} \approx(0.655936,5.04)$ with $\lambda=$ 0.95 and $\mu=1.008$ is depicted in Figure 1 with characteristic direction $\theta=\theta_{2} \approx 0.101084$ which is solved from the characteristic function

$$
\begin{aligned}
\Theta(\theta) \approx & 0.076926 \cos (\theta)^{2}-0.898114 \sin (\theta) \cos (\theta) \\
& +0.014025 \\
= & C_{2}^{(1)}\left(\theta-\theta_{2}\right)+O\left(\left|\theta-\theta_{2}\right|^{2}\right),
\end{aligned}
$$

after we make the polar-coordinate-transformation $x=r \cos (\theta)+x_{*}, y=r \sin (\theta)+y_{*}$. The residual real simple root of equation $\Theta(\theta)=0$ in interval $[0,2 \pi]$ is $\theta_{1} \approx 1.555157$. The power series of above characteristic function $\Theta(\theta)$ up to order two at the point $\theta_{2}$ admits indexes $C_{2}^{(1)}<0$ and $R\left(\theta_{2}\right)<0$ with same negative sign, i.e., $p=1$ (odd number) and $k=2$, where function

$$
\begin{aligned}
R(\theta) \approx & 0.898114 \cos (\theta)^{2}+0.076926 \sin (\theta) \cos (\theta) \\
& -1.438292 .
\end{aligned}
$$

Thus, $\theta=\theta_{2}$ actually shows a fact that trajectories enter the stable node along this direction.

4.3. Nonexistence of Limit Cycles. In this section, we consider nonexistence of closed orbits and limit cycles of system (2a) and system (2b). Firstly, taking a diffeomorphism $\varphi: u=$ $x, v=y, \tau=t /((a+b x+c y)(y+e))$ which preserves the orientation of time, system (2a) and system (2b) are topologically equivalent to following system:

$$
\begin{aligned}
\dot{x}= & P(x, y):=\left[r_{1} x\left(1-\frac{x}{K_{1}}\right)-m_{1} x-\mathrm{d} x^{2}\right] \\
& \cdot(a+b x+c y)(y+e)-q_{1} x y(y+e), \\
\dot{y}= & Q(x, y):=r_{2} y^{2}\left(1-\frac{y}{K_{2}}\right)(a+b x+c y) \\
& +q_{2} x y(y+e)-m_{2} y(a+b x+c y)(y+e),
\end{aligned}
$$

since $\operatorname{det} D \varphi(u, v, \tau)=(v+e)(a+b u+c v)>0, \quad$ where matrix

$$
D \varphi(u, v, \tau)=\left[\begin{array}{ccc}
1 & 0 & 0 \\
0 & 1 & 0 \\
* & * & (v+e)(a+b u+c v)
\end{array}\right] .
$$

Notice that we still denote $u, v$, and $\tau$ as $x, y$, and $t$. Above system is a $C^{\infty}$-qualitatively equivalent polynomial form of system (2a) and system (2b), and it is more convenient to consider limit cycles $[4,5,20,21]$.

Theorem 4 (nonexistence of limit cycles). For system (42a) and system (42b), if parameters satisfy

$$
\begin{aligned}
\left(m_{1}+m_{2}-r_{1}-r_{2}\right) b+c d e+a d-q_{2} & \geq 0, \\
a d+b m_{1}-b r_{1} & \geq 0, \\
(c e+a) m_{2}-a r_{2} & >0, \\
c\left(m_{2}-r_{2}\right) K_{2}+a r_{2} & \geq 0,
\end{aligned}
$$

then there are no closed orbits and limit cycles in $\mathbb{R}^{+2}$.

Proof. Here we take a Dulac function $B(x, y)=1 / x y$ and calculate following partial derivative:

$$
\frac{\partial(B P)}{\partial x}+\frac{\partial(B Q)}{\partial y}=\frac{1}{K_{1} K_{2} x y} \sum_{1 \leq i+j \leq 3} a_{i j} x^{i} y^{j},
$$

where coefficients

$$
\begin{aligned}
a_{02}= & -2 K_{1}\left[c\left(m_{2}-r_{2}\right) K_{2}+a r_{2}\right], \\
a_{11}= & -K_{2}\left[\left(\left(-r_{1}-r_{2}+m_{1}+m_{2}\right) b+c d e+a d-q_{2}\right)\right. \\
& \left.\cdot K_{1}+r_{1}(c e+a)\right], \\
a_{10}=- & -e\left[\left(a d+b m_{1}-b r_{1}\right) K_{1}+a r_{1}\right] K_{2}, \\
a_{01}= & -K_{2}\left[(c e+a) m_{2}-a r_{2}\right] K_{1},
\end{aligned}
$$

and other unlisted coefficients are all nonpositive. Thus, we complete the proof.

Here we take over values of parameters from Section 4.2 but $m_{1}=m_{2}=2$, and system (2a) and system (2b) do not have meaningful equilibria except a globally asymptotically stable node $E_{0}$ (the origin $O$ ) with the characteristic direction $\theta=0$ (the positive $x$-axis) since the characteristic equation $\Theta(\theta)=\left(m_{1}-m_{2}-r_{1}\right) \sin (\theta) \cos (\theta)$, and conditions in Theorem 4 all hold. Hence, there are no closed orbits and limit cycles in this numerical case.

In addition, system (2a) and system (2b) merely own a saddle point $E_{0}$ and a globally asymptotically stable node $E_{1}$ 


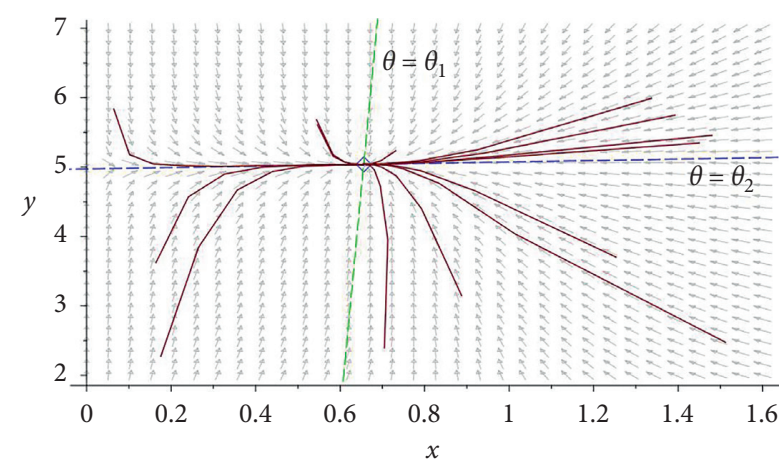

Figure 1: Phase diagrams around an unique stable node $E_{*}$ with characteristic direction $\theta=\theta_{2}$ (in blue).

when we use parameters from Section 4.2 but set $a=2$, $d=3, m_{1}=0.4$, and $m_{2}=1.4$. Conditions in Theorem 4 are also satisfied.

\section{Local Bifurcations of System (2a) and System (2b)}

In this section, we will give sufficient conditions to show the existence of saddle-node bifurcation, transcritical bifurcation, and Hopf bifurcation of system (2a) and system (2b). Firstly, we denote this system in the following form:

$$
\left(\begin{array}{c}
\dot{x} \\
\dot{y}
\end{array}\right)=F(x, y)
$$

for simplicity and convenience.

5.1. Saddle-Node Bifurcation. The two trivial equilibria $E_{2}^{(k)}(k=1,2)$ collide with each other and system (47) has a unique boundary equilibrium $E_{2}$ when $y>0$, if $r_{2}>m_{2}$ and $y_{1}=y_{2}$, or

$$
m_{2}=m_{2}^{[\mathrm{SN}]}:=r_{2}+\frac{2 r_{2} e}{K_{2}}\left(1-\sqrt{1+\frac{K_{2}}{e}}\right) .
$$

Then, there is a chance of bifurcation around this higherorder singular point. Here we choose $m_{2}$ as a bifurcation parameter and select eigenvector $v=\left(\begin{array}{l}0 \\ 1\end{array}\right)$ corresponding to the zero eigenvalue for matrix (30). The eigenvector corresponding to the zero eigenvalue for the transpose of matrix (30) is $w=\left(\begin{array}{c}1 \\ w_{2}\end{array}\right)$, where

$$
w_{2}=-\frac{r_{1}-m_{1}-\left(\left(q_{1} y_{2}\right) /\left(a+c y_{2}\right)\right)}{\left(\left(q_{2} y_{2}\right) /\left(a+c y_{2}\right)\right)} .
$$

Suppose $r_{1}-m_{1}-\left(\left(q_{1} y_{2}\right) /\left(a+c y_{2}\right)\right)<0$, then the following transversality conditions hold:

$$
\begin{gathered}
w^{T} F_{m_{2}}\left(E_{2}, m_{2}^{[\mathrm{SN}]}\right)=-w_{2} y_{2} \neq 0 \\
w^{T}\left[D^{2} F\left(E_{2}, m_{2}^{[\mathrm{SN}]}\right)(v, v)\right]=\frac{2 r_{2} w_{2}}{K_{2}}\left(\sqrt{\frac{e}{K_{2}+e}}-1\right) \neq 0 .
\end{gathered}
$$

Thus, we have following theorem by using Sotomayor's theorem $[22,23]$.

Theorem 5 (saddle-node bifurcation). Suppose that the point $E_{2}$ exists; if $r_{1}-m_{1}-\left(\left(q_{1} y_{2}\right) /\left(a+c y_{2}\right)\right)<0$, then system (47) undergoes a saddle-node bifurcation around point $E_{2}$ with respect to the bifurcation parameter $m_{2}$.

5.2. Transcritical and Pitchfork Bifurcation. The equilibrium $E_{2}^{(1)}$ changes its stability when $r_{1}$ crosses the threshold $r_{1}^{[\mathrm{TC}]}:=m_{1}+\left(\left(q_{1} y_{1}\right) /\left(a+c y_{1}\right)\right)$; in other words, $E_{2}^{(1)}$ is a higher-order equilibrium when $r_{1}=r_{1}^{[\mathrm{TC}]}$. Let $J_{22}\left(E_{2}^{(1)}\right)<0$. Thus, we choose the parameter $r_{1}$ as a bifurcation parameter and an eigenvector $v=\left(\begin{array}{c}1 \\ v_{2}\end{array}\right)$ corresponding to the zero eigenvalue for the Jacobian matrix $J\left(E_{2}^{(1)}\right)$ when $r_{1}=r_{1}^{[\mathrm{TC}]}$, where $v_{2}=-\left(\left(q_{2} y_{1}\right) /\left(J_{22}\left(E_{2}^{(1)}\right)\left(a+c y_{1}\right)\right)\right)>0$. The eigenvector corresponding to the zero eigenvalue for the transpose of matrix $J\left(E_{2}^{(1)}\right)$ is $w=\left(\begin{array}{l}1 \\ 0\end{array}\right)$; then, the transversality conditions are

$$
\begin{aligned}
w^{T} F_{r_{1}}\left(E_{2}^{(1)}, r_{1}^{[\mathrm{TC}]}\right)= & 0, \\
w^{T} D F_{r_{1}}\left(E_{2}^{(1)}, r_{1}^{[\mathrm{TC}]}\right) v= & 1, \\
w^{T} D^{2} F\left(E_{2}^{(1)}, r_{1}^{[\mathrm{TC}]}\right)(v, v)= & \frac{-2 r_{1}^{[\mathrm{TC}]}}{K_{1}}+\frac{2 q_{1} b y_{1}}{\left(a+c y_{1}\right)^{2}}-2 d \\
& +\frac{2 q_{1} q_{2} a y_{1}}{\left(a+c y_{1}\right)^{3} J_{22}\left(E_{2}^{(1)}\right)} .
\end{aligned}
$$

Thus, we have the following theorem by using Sotomayor's theorem $[22,23]$.

Theorem 6 (transcritical bifurcation). Suppose that the two axial equilibria $\left(E_{2}^{(k)}(k=1,2)\right)$ coexist and $J_{22}\left(E_{2}^{(1)}\right)<0$; if $a+c y_{1} \geq b K_{1}$ or $J_{22}\left(E_{2}^{(1)}\right) \geq-\left(\left(q_{2} a\right) /\left(b\left(a+c y_{1}\right)\right)\right)$, then system (47) undergoes a transcritical bifurcation around the point $E_{2}^{(1)}$ with respect to the bifurcation parameter $r_{1}$.

For the special case

$$
w^{T} D^{2} F\left(E_{2}^{(1)}, r_{1}^{[\mathrm{TC}]}\right)(v, v)=0,
$$

there is a chance the system (47) undergoes a pitchfork bifurcation. We still use the bifurcation parameter $r_{1}$ and eigenvectors $v$ and $w$; then, the fourth transversality condition is 


$$
w^{T} D^{3} F\left(E_{2}^{(1)}, r_{1}^{[\mathrm{PF}]}\right)(v, v, v)=-\frac{6 q_{1}\left[b c J_{22} y_{1}+a\left(J_{22} b+q_{2}\right)\right]\left[c\left(J_{22} b-q_{2}\right) y_{1}+a b J_{22}\right] y_{1}}{\left(c y_{1}+a\right)^{5} J_{22}^{2}} \neq 0,
$$

where $\quad J_{22}=J_{22}\left(E_{2}^{(1)}\right), \quad r_{1}^{[\mathrm{PF}]}:=m_{1}+\left(\left(q_{1} y_{1}\right) /\left(a+c y_{1}\right)\right)$. Thus, we have another theorem by using Sotomayor's theorem $[22,23]$.

Theorem 7 (pitchfork bifurcation). Suppose that the two axial equilibria $\left(E_{2}^{(k)}(k=1,2)\right)$ coexist and $J_{22}\left(E_{2}^{(1)}\right)<0$; if condition (52) and $J_{22}\left(E_{2}^{(1)}\right) \neq-\left(\left(a q_{2}\right) /\left(b\left(c y_{1}+a\right)\right)\right)$ hold, then system (47) undergoes a pitchfork bifurcation around the point $E_{2}^{(1)}$ with respect to the bifurcation parameter $r_{1}$.

5.3. Hopf Bifurcation. In this section, we consider the Hopf bifurcation of system (2a) and system (2b). Here the point $E_{*}$ exists and we choose $d$ as bifurcation parameter. Suppose that $\lambda(d)=\alpha(d) \pm i \omega(d)$ are a pair of conjugate eigenvalues of matrix $J\left(E_{*}\right)$, where $\alpha(d)=(1 / 2) A_{1}(d)$. The critical value $d^{[H]}$ satisfies

$$
\begin{gathered}
\alpha\left(d^{[H]}\right)=0, \\
\alpha^{\prime}\left(d^{[H]}\right) \neq 0, \\
A_{2}\left(d^{[H]}\right)>0 .
\end{gathered}
$$

Then, system (47) undergoes a Hopf bifurcation around the point $E_{*}$ with respect to the bifurcation parameter $d$.

We will calculate the first Lyapunov number $\sigma$ at the point $E_{*}$, which is used to determine the stability of limit cycles and Hopf bifurcation direction. The method and calculations of the first and second Lyapunov coefficients can be found in $[22,24,25]$. Therefore, translating the point $E_{*}$ to the origin $O=(0,0)$ by a linear transformation (I): $X=x-x_{*}, Y=y-y_{*}$, system (47) in power series around the origin is

$$
\begin{aligned}
& \dot{X}=f_{1}(X, Y)=\sum_{1 \leq i+j \leq 3} a_{i j} X^{i} Y^{j}+O\left(|X, Y|^{4}\right), \\
& \dot{Y}=g_{1}(X, Y)=\sum_{1 \leq i+j \leq 3} b_{i j} X^{i} Y^{j}+O\left(|X, Y|^{4}\right),
\end{aligned}
$$

where coefficients are

$$
\begin{aligned}
& a_{10}=-\frac{r_{1} s_{1}}{K_{1}}-d s_{1}+\frac{q_{1} b s_{1} s_{2}}{\left(b s_{1}+c s_{2}+a\right)^{2}}, \\
& a_{01}=-\frac{q_{1} s_{1}\left(b s_{1}+a\right)}{\left(b s_{1}+c s_{2}+a\right)^{2}}, \\
& a_{20}=-\frac{r_{1}}{K_{1}}+\frac{q_{1} s_{2} b\left(c s_{2}+a\right)}{\left(b s_{1}+c s_{2}+a\right)^{3}}-d \text {, } \\
& a_{02}=\frac{q_{1} s_{1} c\left(b s_{1}+a\right)}{\left(b s_{1}+c s_{2}+a\right)^{3}}, \\
& a_{11}=-\frac{\left(a^{2}+\left(b s_{1}+c s_{2}\right) a+2 s_{1} s_{2} b c\right) q_{1}}{\left(b s_{1}+c s_{2}+a\right)^{3}}, \\
& a_{30}=-\frac{q_{1} s_{2} b^{2}\left(c s_{2}+a\right)}{\left(b s_{1}+c s_{2}+a\right)^{4}}, \\
& a_{03}=-\frac{q_{1} s_{1} c^{2}\left(b s_{1}+a\right)}{\left(b s_{1}+c s_{2}+a\right)^{4}}, \\
& a_{21}=\frac{b\left(s_{1}\left(2 c s_{2}+a\right) b-c^{2} s_{2}^{2}+a^{2}\right) q_{1}}{\left(b s_{1}+c s_{2}+a\right)^{4}}, \\
& a_{12}=\frac{c\left(s_{2}\left(2 b s_{1}+a\right) c-b^{2} s_{1}^{2}+a^{2}\right) q_{1}}{\left(b s_{1}+c s_{2}+a\right)^{4}} ; \\
& b_{10}=\frac{q_{2} s_{2}\left(c s_{2}+a\right)}{\left(b s_{1}+c s_{2}+a\right)^{2}}, \\
& b_{01}=-\frac{r_{2} s_{2}\left(-K_{2} e+2 e s_{2}+s_{2}^{2}\right)}{K_{2}\left(s_{2}+e\right)^{2}}-\frac{q_{2} c s_{1} s_{2}}{\left(b s_{1}+c s_{2}+a\right)^{2}}, \\
& b_{20}=-\frac{q_{2} s_{2} b\left(c s_{2}+a\right)}{\left(b s_{1}+c s_{2}+a\right)^{3}} \text {, } \\
& b_{02}=\frac{r_{2}\left(\left(K_{2}-3 s_{2}\right) e^{2}-3 s_{2}^{2} e-s_{2}^{3}\right)}{K_{2}\left(s_{2}+e\right)^{3}}-\frac{q_{2} s_{1} c\left(b s_{1}+a\right)}{\left(b s_{1}+c s_{2}+a\right)^{3}}, \\
& b_{11}=\frac{\left(a^{2}+\left(b s_{1}+c s_{2}\right) a+2 s_{1} s_{2} b c\right) q_{2}}{\left(b s_{1}+c s_{2}+a\right)^{3}} \text {, }
\end{aligned}
$$




$$
\begin{aligned}
& b_{30}=\frac{q_{2} s_{2} b^{2}\left(c s_{2}+a\right)}{\left(b s_{1}+c s_{2}+a\right)}, \\
& b_{03}=-\frac{r_{2} e^{2}\left(K_{2}+e\right)}{K_{2}\left(s_{2}+e\right)^{4}}+\frac{q_{2} s_{1} c^{2}\left(b s_{1}+a\right)}{\left(b s_{1}+c s_{2}+a\right)^{4}}, \\
& b_{21}=-\frac{b\left(s_{1}\left(2 c s_{2}+a\right) b-c^{2} s_{2}^{2}+a^{2}\right) q_{2}}{\left(b s_{1}+c s_{2}+a\right)^{4}}, \\
& b_{12}=-\frac{c\left(s_{2}\left(2 b s_{1}+a\right) c-b^{2} s_{1}^{2}+a^{2}\right) q_{2}}{\left(b s_{1}+c s_{2}+a\right)^{4}}
\end{aligned}
$$

and $O\left(|X, Y|^{4}\right)$ stands for some smooth functions. After that, by using a transformation (II): $u=Y, v=\left(-X b_{10}+Y a_{10}\right) / \beta$ with $\beta=\sqrt{A_{2}\left(E_{*}\right)}>0$, the above system becomes a standard form:

$$
\begin{aligned}
& \dot{u}=f_{2}(u, v)=-\beta v+\sum_{2 \leq i+j \leq 3} A_{i j} u^{i} v^{j}+O\left(|u, v|^{4}\right), \\
& \dot{v}=g_{2}(u, v)=\beta u+\sum_{2 \leq i+j \leq 3} B_{i j} u^{i} v^{j}+O\left(|u, v|^{4}\right),
\end{aligned}
$$

where

$$
\begin{aligned}
& A_{20}=\frac{\left(a_{10}^{2} b_{20}+a_{10} b_{10} b_{11}+b_{02} b_{10}^{2}\right)}{b_{10}^{2}}, \\
& A_{11}=\frac{-\left(2 a_{10} b_{20}+b_{10} b_{11}\right) \beta}{b_{10}^{2}}, \\
& A_{02}=\frac{-b_{20}\left(a_{01} b_{10}+a_{10}^{2}\right)}{b_{10}^{2}} \\
& A_{30}=\frac{a_{10}^{3} b_{30}+a_{10}^{2} b_{10} b_{21}+a_{10} b_{10}^{2} b_{12}+b_{03} b_{10}^{3}}{b_{10}^{3}} \text {, } \\
& A_{21}=\frac{-\left(3 a_{10}^{2} b_{30}+2 a_{10} b_{10} b_{21}+b_{10}^{2} b_{12}\right) \beta}{b_{10}^{3}}, \\
& A_{12}=\frac{-\left(3 a_{10} b_{30}+b_{10} b_{21}\right)\left(a_{01} b_{10}+a_{10}^{2}\right)}{b_{10}^{3}}, \\
& A_{03}=\frac{-b_{30} \beta^{3}}{b_{10}^{3}} \\
& B_{20}=-\frac{a_{02} b_{10}^{3}-a_{10}^{3} b_{20}+a_{10}^{2} a_{20} b_{10}-a_{10}^{2} b_{10} b_{11}+a_{10} a_{11} b_{10}^{2}-a_{10} b_{02} b_{10}^{2}}{\beta b_{10}^{2}}, \\
& B_{11}=-\frac{2 a_{10}^{2} b_{20}-2 a_{10} a_{20} b_{10}+a_{10} b_{10} b_{11}-a_{11} b_{10}^{2}}{b_{10}^{2}} \\
& B_{02}=\frac{\left(a_{10} b_{20}-a_{20} b_{10}\right) \beta}{b_{10}^{2}} \\
& B_{30}=-\frac{a_{03} b_{10}^{4}-a_{10}^{4} b_{30}+a_{10}^{3} a_{30} b_{10}-a_{10}^{3} b_{10} b_{21}+a_{10}^{2} a_{21} b_{10}^{2}-a_{10}^{2} b_{10}^{2} b_{12}+a_{10} a_{12} b_{10}^{3}-a_{10} b_{03} b_{10}^{3}}{\beta b_{10}^{3}}, \\
& B_{21}=-\frac{3 a_{10}^{3} b_{30}-3 a_{10}^{2} a_{30} b_{10}+2 a_{10}^{2} b_{10} b_{21}-2 a_{10} a_{21} b_{10}^{2}+a_{10} b_{10}^{2} b_{12}-a_{12} b_{10}^{3}}{b_{10}^{3}}, \\
& B_{12}=\frac{\beta\left(3 a_{10}^{2} b_{30}-3 a_{10} a_{30} b_{10}+a_{10} b_{10} b_{21}-a_{21} b_{10}^{2}\right)}{b_{10}^{3}}, \\
& B_{03}=\frac{\left(a_{10} b_{30}-a_{30} b_{10}\right)\left(a_{01} b_{10}+a_{10}^{2}\right)}{b_{10}^{3}} \text {. }
\end{aligned}
$$


Let $p=q=(1 / \sqrt{2})\left(\begin{array}{l}i \\ 1\end{array}\right)$ be two corresponding eigenvectors of a matrix $A$ such that $A q=i \beta q, A^{\top} p=-i \beta p$ and $\langle p, q\rangle=1$; the operation $\langle x, y\rangle=x^{\dagger} y(x, y \in \mathbb{C})$ with the Hermitian transpose (upper symbol) $\dagger$ represents the usual inner product, and the Jacobian matrix is

$$
A=\left[\begin{array}{cc}
0 & -\beta \\
\beta & 0
\end{array}\right] \text {. }
$$

We should rewrite the functions in the right hand side of system (57) in the form of power series

$$
F_{2}(x)=\left(\begin{array}{l}
f_{2}(x) \\
g_{2}(x)
\end{array}\right)=A x+\frac{1}{2 !} B(x, x)+\frac{1}{3 !} C(x, x, x)+O\left(\|x\|^{4}\right),
$$

where the components of linear functions $B$ and $C$ are

$$
\begin{aligned}
B_{i}(x, y) & =\sum_{j, k=1}^{2} \frac{\partial^{2}\left(F_{2}\right)_{i}(0)}{\partial \xi_{j} \partial \xi_{k}} x_{j} y_{k}, \\
C_{i}(x, y, z) & =\sum_{j, k, l=1}^{2} \frac{\partial^{3}\left(F_{2}\right)_{i}(0)}{\partial \xi_{j} \partial \xi_{k} \partial \xi_{l}} x_{j} y_{k} z_{l}, \quad i=1,2,
\end{aligned}
$$

while $\|x\|$ is the two-dimensional Euclidean norm of $x$. Define $T^{c}$ be the largest subspace invariant by the matrix $A$ and the generalized eigen subspace corresponding to the pair of purely imaginary eigenvalues $\pm i \beta$, i.e., $T^{c}=\operatorname{span}\{q, \bar{q}\}$. That is to say, for any element $z \in T^{c}$, there must exist a linear expansion $z=w q+\overline{w q}$. Now we can construct a twodimensional parameterized center manifold

$$
H=H(w, \bar{w})=w q+\overline{w q}+\sum_{j+k \geq 2}^{\infty} \frac{1}{j ! \cdot k !} h_{j k} w^{j} \bar{w}^{k},
$$

in which $h_{j k} \in \mathbb{C}^{2}$ and its complex conjugate is $\overline{h_{j k}}=h_{k j}$. Combining equations (57) and $H(w, \bar{w})$, we arrive at a complex equation without consideration:

$$
\frac{\partial H}{\partial w} \dot{w}+\frac{\partial H}{\partial \bar{w}} \dot{\bar{w}}=F_{2}(H) .
$$

The "chart" $w$ for the central manifold $H$ should be extracted from following differential equation:

$$
\dot{w}=i \beta w+\frac{1}{2} G_{21} w|w|^{2}+\cdots
$$

So, substituting it into equation (63) and comparing coefficients of these $w^{j} \bar{w}^{k}$, we recursively derive

$$
h_{11}=-A^{-1} B(q, \bar{q}), h_{20}=(2 i \beta I-A)^{-1} B(q, q),
$$

and an equality from coefficient of the cubic term $w|w|^{2}$ :

$$
(i \beta I-A) h_{21}+G_{21} q=B\left(\bar{q}, h_{20}\right)+2 B\left(q, h_{11}\right)+C(q, q, \bar{q}) .
$$

Here letter $I$ represents the unit matrix with rank 2. It is quite apparent that equality (66) admits a solution:

$$
G_{21}=\left\langle p, B\left(\bar{q}, h_{20}\right)+2 B\left(q, h_{11}\right)+C(q, q, \bar{q})\right\rangle .
$$

At last, the first Lyapunov coefficient is calculated as

$$
\begin{aligned}
l_{1}= & \frac{1}{2} \operatorname{Re}\left(G_{21}\right) \\
= & \frac{1}{4 \beta}\left[\left(B_{21}+A_{12}+3 A_{30}+3 B_{03}\right) \beta+\left(A_{11}+2 B_{02}\right) A_{02}\right. \\
& \left.+A_{11} A_{20}-2 A_{20} B_{20}-B_{11}\left(B_{02}+B_{20}\right)\right] .
\end{aligned}
$$

Thus, the first Lyapunov number $\sigma$ for the focus of planar system (57) is given by the formula $\sigma=(6 \pi / \beta) l_{1}$. Since above expression is much too complicated, we need to present some numerical simulations and figures around the point $E_{*}$ with computer simulations.

Theorem 8 (nondegenerate Hopf bifurcation). Assume that the equilibrium $E_{*}$ exists and parameters satisfy condition (54) and $\sigma \neq 0$; then, system (47) undergoes a nondegenerate Hopf bifurcation around this equilibrium as parameter $\delta=d$ passes through the critical value $d^{[H]}$. The Hopf bifurcation is supercritical (subcritical), the interior equilibrium $E_{*}$ is a multiple stable (unstable) focus with multiplicity one, and limit cycles are stable (unstable) if $\sigma<0(\sigma>0)$.

Remark 2. The first Lyapunov coefficient $l_{1}$ can be defined by $(1 / 2 \beta) \operatorname{Re}\left(G_{21}\right)$ at times. On occasion, there are times when our system (55) exists some values of parameters such that $\sigma=0$ or the system may undergoes a degenerate Hopf bifurcation. Accompanied by a proper transformation, for planar system (55), the first Lyapunov number is given by the following formula [22]:

$$
\begin{aligned}
\sigma_{1}= & -\frac{3 \pi}{2 a_{01} A_{2}^{3 / 2}}\left\{\left[a_{10} b_{10}\left(a_{11}^{2}+a_{11} b_{02}+a_{02} b_{11}\right)+a_{10} a_{01}\left(b_{11}^{2}+a_{20} b_{11}+a_{11} b_{20}\right)\right.\right. \\
& +b_{10}^{2}\left(a_{11} a_{02}+2 a_{02} b_{02}\right)-2 a_{10} b_{10}\left(b_{02}^{2}-a_{20} a_{02}\right)-2 a_{10} a_{01}\left(a_{20}^{2}-b_{20} b_{02}\right) \\
& \left.-a_{01}^{2}\left(2 a_{20} b_{20}+b_{11} b_{20}\right)+\left(a_{01} b_{10}-2 a_{10}^{2}\right)\left(b_{11} b_{02}-a_{11} a_{20}\right)\right]-\left(a_{10}^{2}+a_{01} b_{10}\right)\left[3\left(b_{10} b_{03}-a_{01} a_{30}\right)\right. \\
& \left.\left.+2 a_{10}\left(a_{21}+b_{12}\right)+\left(b_{10} a_{12}-a_{01} b_{21}\right)\right]\right\}
\end{aligned}
$$




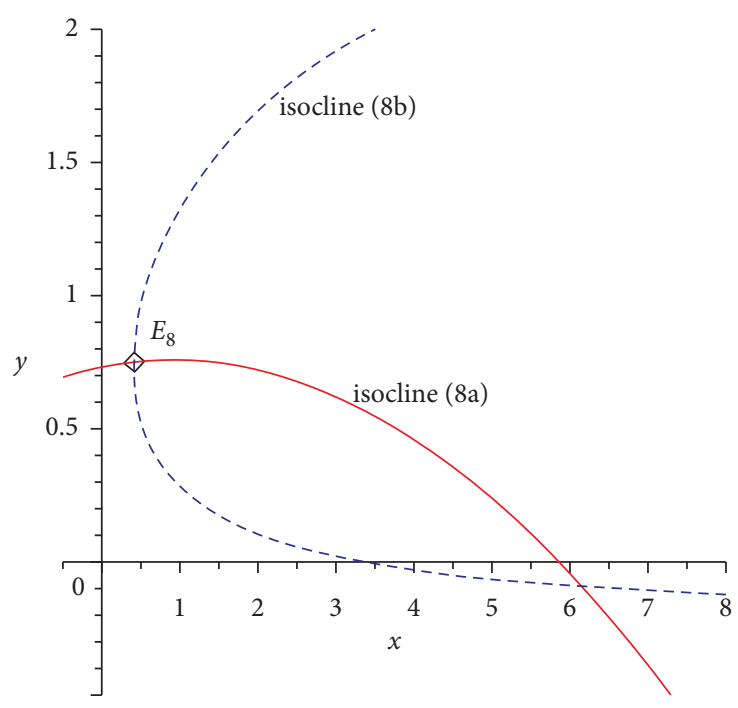

(a)

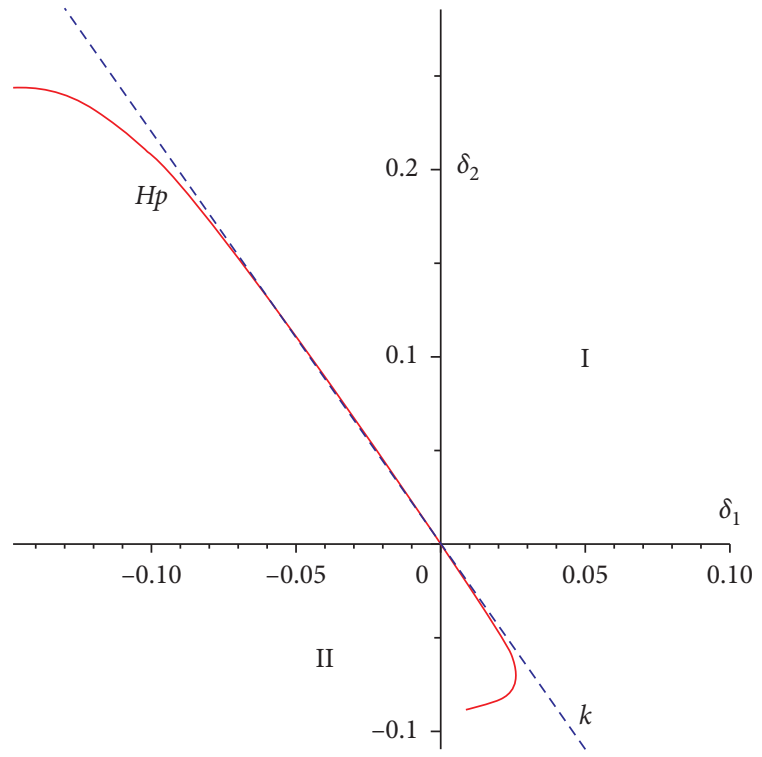

(b)

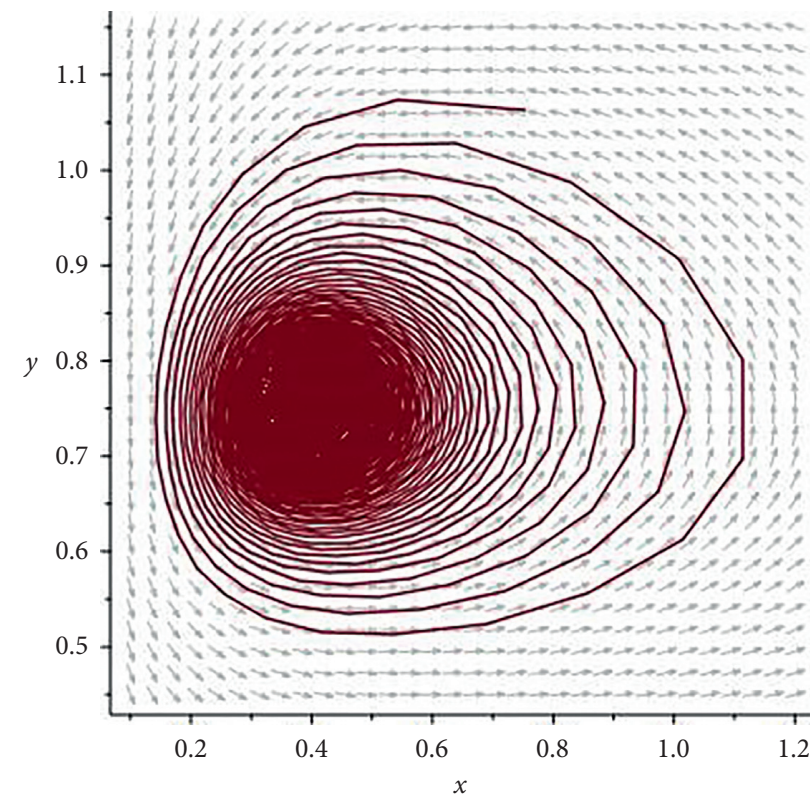

(c)

Figure 2: Figures in Example 1. (a) Existence of interior equilibrium $E_{8}$ in Case 6: red isocline (10a) and blue isocline (10b). (b) Hopf bifurcation curve Hp (in red). (c) A stable focus with $d=0.0595$.

which is an advanced version of $\sigma$ or $l_{1}$.

Finally, for the Hopf bifurcation, we numerically give following examples to simulate how the parameter $d$ controls dynamical behavior of system (2a) and system (2b).

Example 1. Consider Case 6 in Section 3.1 and Theorem 4 again. Figure 2(a) reveals the existence of this interior equilibrium $E_{8}$ in Case 6 by using the cobweb method. To investigate how the control parameter $d$ affects dynamical behavior of our system (2a) and system (2b), Figures 2(c) and 3 depict phase diagrams corresponding to values $d=0.0595$ and $d=0.053$, respectively. When $d=0.0595$, this interior equilibrium is an asymptotically stable focus since $A_{1} \approx-0.00111352489, \quad A_{2} \approx 0.07340334276$, and $\Delta_{*} \approx$ -0.2936121311 . When $d=0.053$, it gets $A_{1} \approx 0.00017832135$, $A_{2} \approx 0.07359407961$, and $\Delta_{*} \approx-0.2943762866$, and there exists a limit cycle (by using the Poincare-Bendixson theorem) around this unstable focus. Here we notice that

$$
\alpha\left(d_{1}\right)-\alpha\left(d_{2}\right) \approx \alpha^{\prime}\left(d_{12}^{[H]}\right)\left(d_{1}-d_{2}\right)+o\left(10^{-6}\right) .
$$

This implies that the Hopf bifurcation occurs in system (2a) and system (2b) when $d=d_{8}^{[H]}$. The first Lyapunov coefficient $l_{1} \approx-0.122574$, and thus the Hopf bifurcation is supercritical and a limit cycle generated by the critical point 


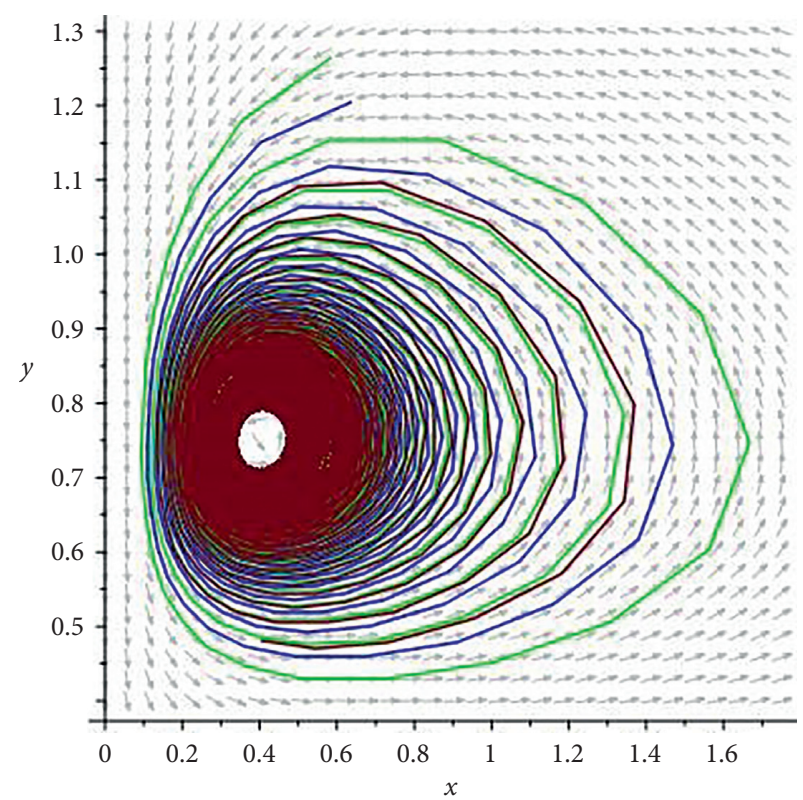

(a)

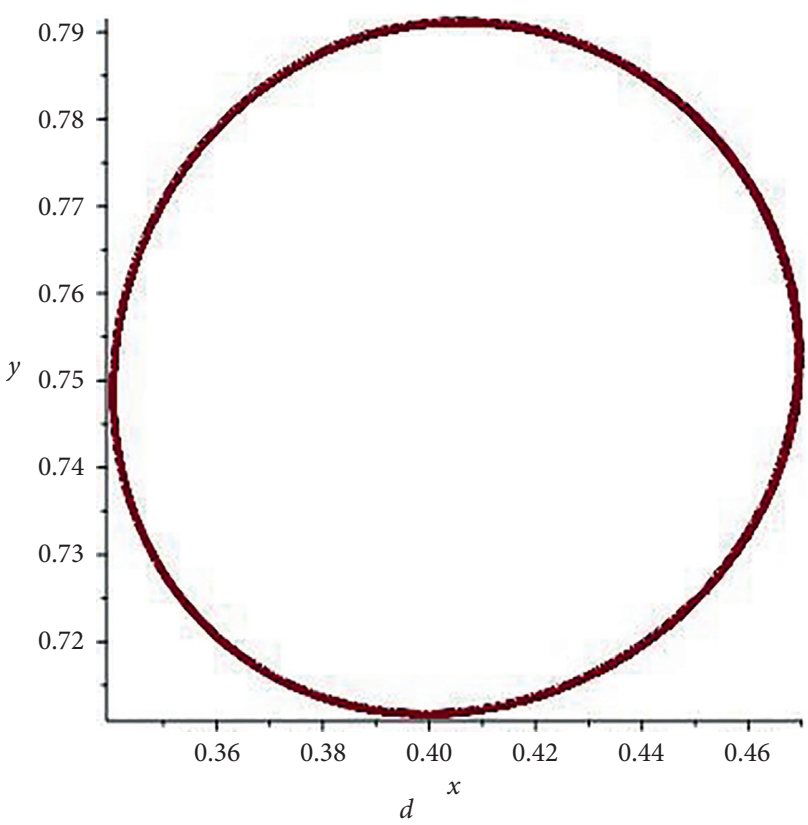

(b)

Figure 3: Figures in Example 1 with $d=0.053$. (a) Phase diagrams around an unstable focus. (b) Enlarged phase diagrams: limit cycle.

is stable. This interior equilibrium is a multiple stable focus with multiplicity one.

Besides, for a perturbed system with sufficiently small parameter vector $\delta=\left(\delta_{1}, \delta_{2}\right)$ in a neighbourhood of the origin $O$ in the parameter plane

$\dot{x}=r_{1} x\left(1-\frac{x}{K_{1}}\right)-\frac{q_{1} x y}{a+b x+c y}-m_{1} x-\left(d+\delta_{1}\right) x^{2}$,

$\dot{y}=r_{2} y\left(1-\frac{y}{K_{2}}\right) \frac{y}{y+e}+\frac{q_{2} x y}{a+b x+c y}-\left(m_{2}+\delta_{2}\right) y$,

and Hopf bifurcation analysis with two bifurcation parameters $d$ and $m_{2}$, we let $\delta \neq 0$ and suppose that $E_{*}=\left(x_{*}, y_{*}\right)$ is an interior equilibrium of above perturbed system, where $y_{*}=y_{8}+w,|w|$ is sufficiently small, and

$x_{*}=-\frac{\left(c y_{*}+a\right)\left\{\left[\left(\delta_{2}+m_{2}-r_{2}\right) y_{*}+e\left(m_{2}+\delta_{2}\right)\right] K_{2}+r_{2} y_{*}^{2}\right\}}{\left\{\left[\left(\delta_{2}+m_{2}-r_{2}\right) b-q_{2}\right] y_{*}+e\left[\left(m_{2}+\delta_{2}\right) b-q_{2}\right]\right\} K_{2}+b r_{2} y_{*}^{2}}$.

Substituting it into $A_{1}$ and $A_{2}$, the solutions $\delta_{1}=\delta_{1}(w)$ and $\delta_{2}=\delta_{2}(w)$ can be directly solved, which are written as the form up to third order:

$$
\begin{aligned}
& \delta_{1} \approx-1.503836 w+24.893383 w^{2}+O\left(w^{3}\right), \\
& \delta_{2} \approx 3.309615 w-56.757836 w^{2}+O\left(w^{3}\right),
\end{aligned}
$$

and hence the slope $k=\lim _{w \longrightarrow 0}\left(\left(\delta_{2}(w)\right) /\left(\delta_{1}(w)\right)\right)$ of approximation straight line of the Hopf bifurcation curve Hp (see Figure 2(b)) in a small neighbourhood of the origin in parameter plane is approximately about -2.200781 . The supercritical Hopf bifurcation curve $H p$ of system (71a) and system $(71 \mathrm{~b})$ at the point $E_{8}$ is numerically defined by

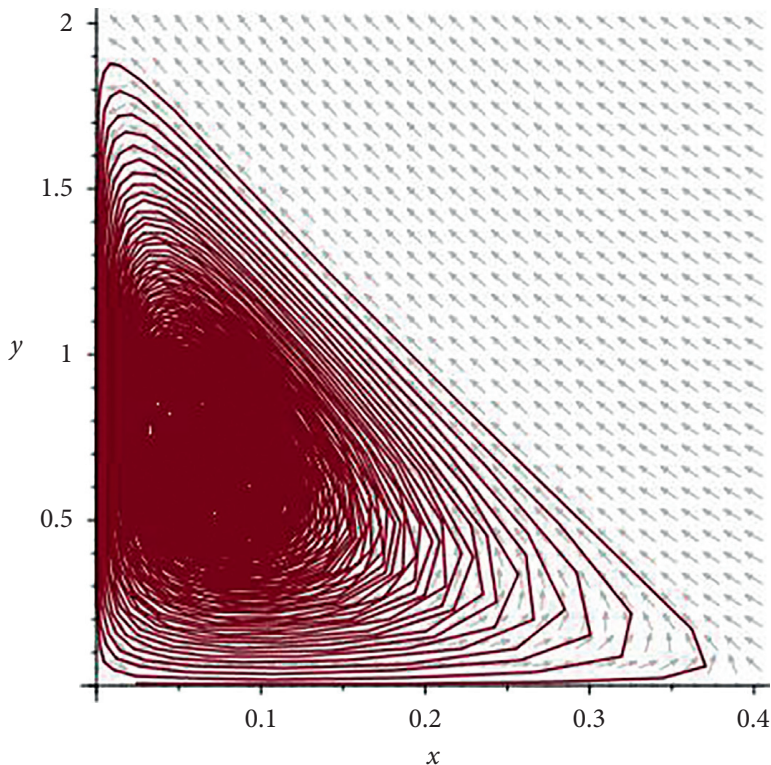

Figure 4: A stable focus in Example 2 with $d=2.109$.

solution (73), i.e., $H p=\{\delta \mid \delta t n$ satisfies $q(73)\}$, while the variables $\delta$ and $w$ both ensure the existence of $E_{*}$ and

$$
A_{2}(w) \approx 0.073568+2.087448 w-47.606989 w^{2}+O\left(w^{3}\right)>0 .
$$

As a matter of fact, we perceive the phenomenon that Theorem 8 describes the special case once $\delta$ lies on the horizontal line $\delta_{2}=0$.

Example 2. Here we set parameters as well as Case 3 in Section 3.2. Figures 4 and 5 depict phase diagrams 


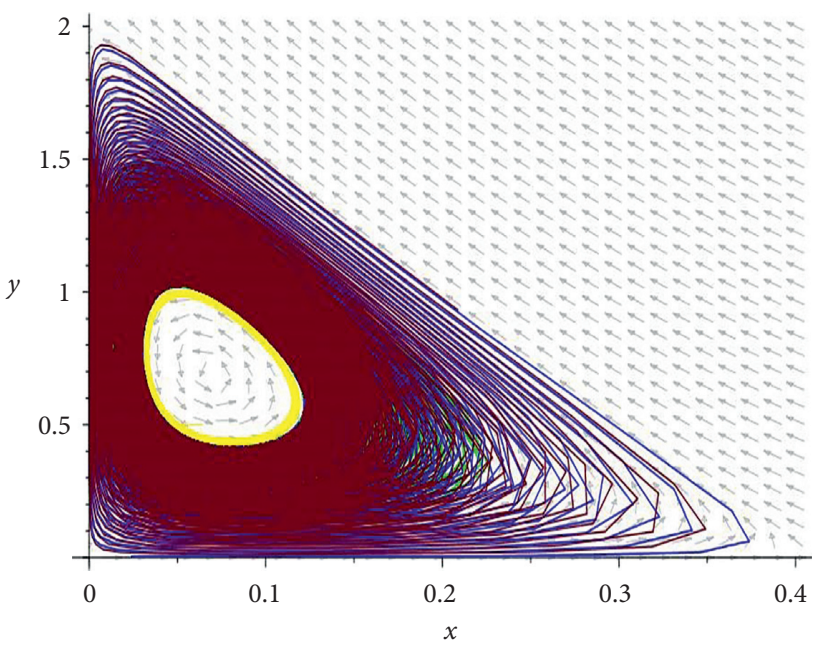

(a)

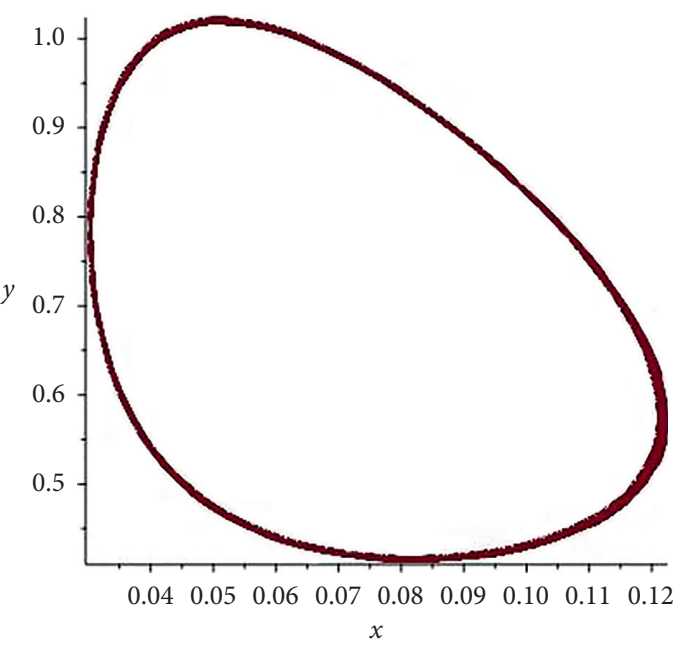

(b)

Figure 5: Figures in Example 2 with $d=2.09$. (a) Phase diagrams around an unstable focus. (b) Enlarged phase diagrams: limit cycle.

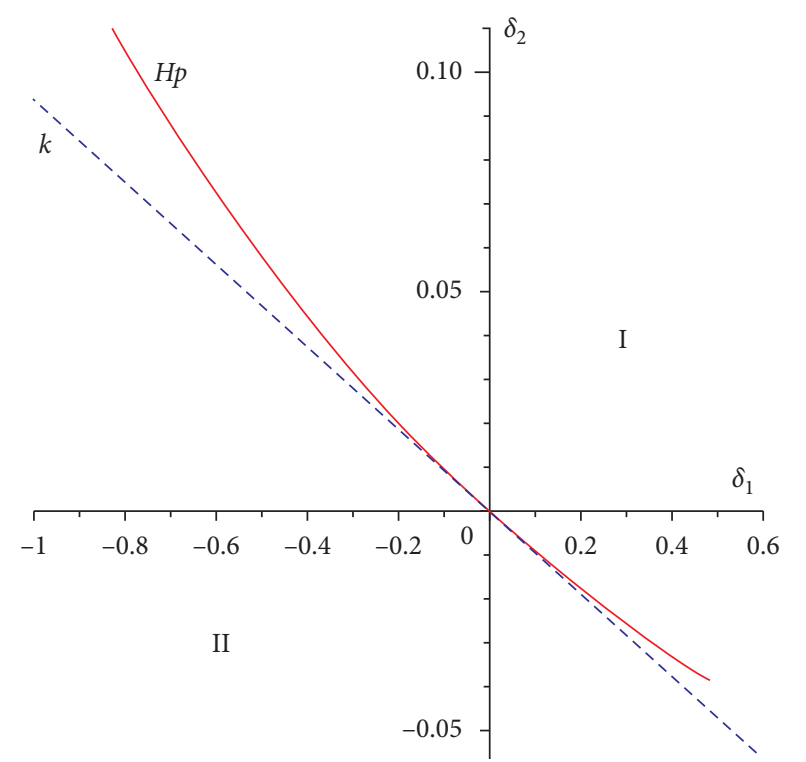

FIgURE 6: Hopf bifurcation curve Hp (in red) in Example 2.

corresponding to values $d_{1}=2.109$ and $d_{2}=2.09$, respectively. One should notice that

$$
\alpha\left(d_{1}\right)-\alpha\left(d_{2}\right) \approx \alpha^{\prime}\left(d_{12}^{[H]}\right)\left(d_{1}-d_{2}\right)+o\left(10^{-6}\right) .
$$

Similar to above example, system (2a) and system (2b) undergo a Hopf bifurcation when $d$ passes through $d_{12}^{[H]}$. The first Lyapunov coefficient $l_{1} \approx-0.002729$ is also found to be negative. Thus, the Hopf bifurcation is supercritical and a limit cycle generated by the critical point is stable. The interior equilibrium is also a multiple stable focus with multiplicity one. Figure 6 is the Hopf bifurcation curve corresponding to system (69) with slope $k \approx-0.094044$ and

$$
\begin{aligned}
H p=\left\{\delta \mid \delta_{1} \approx-40.550983 w+639.229323 w^{2}\right. \\
\\
\left.+O\left(w^{3}\right), \delta_{2} \approx 3.813575 w-4.617377 w^{2}+O\left(w^{3}\right)\right\} .
\end{aligned}
$$

\section{Conclusions}

In summary, we firstly considered stability analysis and bifurcations of system (2a) and system (2b) with B-D functional response and Allee-like effect, which is a modified version of a predator-prey system in [1]. The polynomial's method, derived from eliminants $R_{y}(f, g)$ and $R_{x}(f, g)$, can be extended to more complicated polynomial systems. Some conclusions are the same as reference [1], such as the uniform boundedness (Theorem $1)$, the existence of equilibria $E_{*}$, the nonexistence (Theorem 4) of limit cycles, and so on. It is supposed that some methods and conclusions can be available in original system (1a) and system (1b), such as pitchfork bifurcation. Lemmas 1 and 2 are available in more complicated predator-prey systems. Some critical cases, such as $r_{1}-$ $m_{1}=\left(\left(q_{1} y_{1}\right) /\left(a+c y_{1}\right)\right)$ and $A_{2}=0$, need to be researched further with the help of topologically equivalent systems or the "blow-up" method (horizontal and vertical blowups). Other parameters can also be considered as a bifurcation parameter $\delta$ in Hopf bifurcation, although it is described by Hopf bifurcation curve $\mathrm{Hp}$, for instance $\delta=x_{*}$. Notice that Theorem 2 (permanence) can be extended to its reaction-diffusive version $[26,27]$. Under the conditions or the discussion of Theorem 3, the Turing instability of its corresponding reaction-diffusion system subject to the homogeneous Neumann boundary conditions [28]:

$$
\begin{gathered}
u_{t}-D_{1} u_{x x}=r_{1} u\left(1-\frac{u}{K_{1}}\right)-\frac{q_{1} u v}{a+b u+c v}-m_{1} u-\mathrm{d} u^{2}, \\
v_{t}-D_{2} v_{x x}=r_{2} v\left(1-\frac{v}{K_{2}}\right) \frac{v}{v+e}+\frac{q_{2} u v}{a+b u+c v}-m_{2} v
\end{gathered}
$$

$$
\partial_{\nu} u=\partial_{\nu} v=0, \quad t \geq 0, x \in \partial(\Omega),
$$




$$
\begin{array}{r}
u(0, x)=u_{0}(x) \geq 0(\neq 0), \\
v(0, x)=v_{0}(x) \geq 0(\neq 0), \\
x \in \Omega,
\end{array}
$$

will not occur, and thus it is available to find potential Hopf bifurcation points and consider transversality conditions if we choose $u_{*}$ as Hopf bifurcation parameter. Here $D_{1}$ and $D_{2}$ are two positive diffusive constants; $\Delta$ is the Laplacian operator; $u=u(t, x)$ and $v=v(t, x)$ are the densities of prey and predator, respectively; $\Omega$ is an one-dimensional bounded domain with smooth boundary $\partial(\Omega)$; the symbol $\partial_{\gamma}$ is the outer flux, and no flux boundary condition is imposed; thus, the system is closed [29]; the admissible initial functions $u_{0}(x)$ and $v_{0}(x)$ are all continuous functions on $\bar{\Omega}$; to describe an environment surrounded by dispersal barriers, we take zero flux at $\partial(\Omega)$ [28]. The method of calculating the first Lyapunov coefficient $l_{1}$ in Section 5.3 is a reference for deducing of Hopf bifurcation direction in reaction-diffusion systems $[29,30]$.

Though the dynamical behavior of predator-prey systems in single species or multispecies has been researched by many previous literatures, we still need further study in biomathematics, especially the phytoplankton and zooplankton systems. Meanwhile, how to keep ecosystems in balance or coexistence states and avoid harmful effect is our next direction in this area.

\section{Appendix}

\section{A. Polynomials $p(x)$ and $q(y)$}

The polynomial $p(x)$ in Section 3.2 is $p(x)=\sum_{k=0}^{5} a_{k} x^{k}$, where coefficients are

$$
\begin{aligned}
& a_{5}=b d^{2}\left(K_{2} c^{2} d q_{2}+b^{2} q_{1} r_{2}\right) K_{1}^{3}-2\left(-q_{1} r_{2} b^{3} d-\frac{3}{2} q_{2} b c^{2} d^{2} K_{2}\right) r_{1} K_{1}^{2}+b\left(3 K_{2} c^{2} d q_{2}+b^{2} q_{1} r_{2}\right) r_{1}^{2} K_{1}+q_{2} r_{1}^{3} c^{2} K_{2} b \\
& a_{4}=3\left(\frac{1}{3} c\left(-\left(\left(m_{2}-r_{2}\right) b-2 q_{2}\right) b q_{1}+q_{2} c\left(-c d e+a d+3 b m_{1}-3 b r_{1}\right)\right) d K_{2}+q_{1} r_{2} b^{2}\left(a d-\frac{2}{3} r_{1} b+\frac{2}{3} b m_{1}\right)\right) \\
& \cdot d K_{1}^{3}-2\left(\left(-\frac{3}{2}\left(-\frac{2}{3}\left(\left(m_{2}-r_{2}\right) b-2 q_{2}\right) b q_{1}+q_{2} c\left(-c d e+a d+2 b m_{1}-2 b r_{1}\right)\right) c d K_{2}-3\left(a d-\frac{1}{3} r_{1} b+\frac{1}{3} b m_{1}\right) r_{2} b^{2} q_{1}\right) r_{1} K_{1}^{2}\right. \\
& +\left(3\left(-\frac{1}{3}\left(\left(m_{2}-r_{2}\right) b-2 q_{2}\right) b q_{1}+q_{2} c\left(-c d e+a d+b m_{1}-b r_{1}\right)\right) c K_{2}+3 a q_{1} r_{2} b^{2}\right) r_{1}^{2} K_{1}+q_{2} r_{1}^{3} c^{2} K_{2}(-c e+a), \\
& a_{3}=\left(-2\left(\frac{1}{2}\left(\left(m_{2}-r_{2}\right) b-q_{2}\right) b q_{1}^{2}+c\left(-\frac{1}{2} d e\left(b m_{2}-3 q_{2}\right) c-\left(\left(m_{2}-r_{2}\right) b-2 q_{2}\right) b r_{1}\right.\right.\right. \\
& \left.\left.+\left(\left(m_{2}-r_{2}\right) b-q_{2}\right) d a+\left(\left(m_{2}-r_{2}\right) b-2 q_{2}\right) m_{1} b\right) q_{1}+\frac{3}{2} q_{2} c^{2}\left(r_{1}-m_{1}\right)\left(-c d e+a d+b m_{1}-b r_{1}\right)\right) d K_{2} \\
& \left.+3\left(\frac{1}{3} r_{1}^{2} b^{2}+\left(-2 a b d-\frac{2}{3} b^{2} m_{1}\right) r_{1}+a^{2} d^{2}+2 a b d m_{1}+\frac{1}{3} b^{2} m_{1}^{2}\right) r_{2} b q_{1}\right) K_{1}^{3} \\
& -2\left(\left(\frac{1}{2}\left(\left(m_{2}-r_{2}\right) b-q_{2}\right) b q_{1}^{2}+2 c\left(-\frac{1}{2} d e\left(b m_{2}-3 q_{2}\right) c-\frac{1}{2}\left(\left(m_{2}-r_{2}\right) b-2 q_{2}\right) b r_{1}\right.\right.\right. \\
& \left.+\left(\left(m_{2}-r_{2}\right) b-q_{2}\right) d a+\frac{1}{2}\left(\left(m_{2}-r_{2}\right) b-2 q_{2}\right) m_{1} b\right) q_{1}+3 c^{2} q_{2}\left(r_{1}-m_{1}\right)\left(-c d e+a d-\frac{1}{2} r_{1} b\right. \\
& \left.\left.\left.+\frac{1}{2} b m_{1}\right)\right) K_{2}-3 a q_{1} r_{2} b\left(a d+b m_{1}-b r_{1}\right)\right) r_{1} K_{1}^{2}+\left(-2\left(\left(-\frac{1}{2} e\left(b m_{2}-3 q_{2}\right) c\right.\right.\right. \\
& \left.\left.\left.+\left(\left(m_{2}-r_{2}\right) b-q_{2}\right) a\right) q_{1}+\frac{3}{2} q_{2} c\left(r_{1}-m_{1}\right)(-c e+a)\right) c K_{2}+3 a^{2} q_{1} r_{2} b\right) r_{1}^{2} K_{1}, \\
& \cdot a_{2}=\left(\left(\left(2\left(m_{2} b-\frac{3}{2} q_{2}\right) e d c+\left(\left(m_{2}-r_{2}\right) b-q_{2}\right) b r_{1}-2\left(\left(m_{2}-r_{2}\right) b-\frac{1}{2} q_{2}\right) d a\right.\right.\right. \\
& \left.-\left(\left(m_{2}-r_{2}\right) b-q_{2}\right) m_{1} b\right) q_{1}^{2}-c\left(-e\left(\left(-2 b m_{2}+6 q_{2}\right) r_{1}+a m_{2} d+2 m_{1}\left(b m_{2}-3 q_{2}\right)\right) d c\right. \\
& +\left(\left(m_{2}-r_{2}\right) b-2 q_{2}\right) b r_{1}^{2}+\left(-4\left(\left(m_{2}-r_{2}\right) b-q_{2}\right) d a-\left(2\left(\left(m_{2}-r_{2}\right) b-2 q_{2}\right) m_{1} b\right) r_{1}\right.
\end{aligned}
$$




$$
\begin{aligned}
& \left.+d^{2}\left(m_{2}-r_{2}\right) a^{2}+4\left(\left(m_{2}-r_{2}\right) b-q_{2}\right) m_{1} d a+\left(\left(m_{2}-r_{2}\right) b-2 q_{2}\right) m_{1}^{2} b\right) q_{1} \\
& \left.+3\left(-d e c+a d-\frac{1}{3} r_{1} b+\frac{1}{3} b m_{1}\right) c^{2} q_{2}\left(r_{1}-m_{1}\right)^{2}\right) K_{2}+a r_{2} q_{1}\left(3 r_{1}^{2} b^{2}+\left(-6 a b d-6 b^{2} m_{1}\right) r_{1}\right. \\
& \left.\left.+a^{2} d^{2}+6 a b d m_{1}+3 b^{2} m_{1}^{2}\right)\right) K_{1}^{3}-2\left(\left(\left(-\left(m_{2} b-\frac{3}{2} q_{2}\right) e c+a\left(\left(m_{2}-r_{2}\right) b\right.\right.\right.\right. \\
& \left.\left.-\frac{1}{2} q_{2}\right)\right) q_{1}^{2}+c\left(-e\left(\left(-b m_{2}+3 q_{2}\right) r_{1}+a m_{2} d+m_{1}\left(b m_{2}-3 q_{2}\right)\right) c+a\left(\left(\left(-2 m_{2}+2 r_{2}\right) b\right.\right.\right. \\
& \left.\left.\left.\left.+2 q_{2}\right) r_{1}+d\left(m_{2}-r_{2}\right) a+2\left(\left(m_{2}-r_{2}\right) b-q_{2}\right) m_{1}\right)\right) q_{1}-\frac{3}{2} q_{2} c^{2}\left(r_{1}-m_{1}\right)^{2}(-c e+a)\right) K_{2} \\
& \left.-a^{2} q_{1} r_{2}\left(a d+3 b m_{1}-3 b r_{1}\right)\right) r_{1} K_{1}^{2}+a\left(-c\left(-m_{2} e c+a\left(m_{2}-r_{2}\right)\right) K_{2}+a^{2} r_{2}\right) q_{1} r_{1}^{2} K_{1} \text {, } \\
& a_{1}=\left(\left(e\left(b m_{2}-q_{2}\right) q_{1}^{3}+\left(2 e\left(\left(-m_{2} b+\frac{3}{2} q_{2}\right) r_{1}+a m_{2} d+m_{1}\left(m_{2} b-\frac{3}{2} q_{2}\right)\right) c\right.\right.\right. \\
& \left.-a\left(\left(\left(-2 m_{2}+2 r_{2}\right) b+q_{2}\right) r_{1}+d\left(m_{2}-r_{2}\right) a+2\left(\left(m_{2}-r_{2}\right) b-\frac{1}{2} q_{2}\right) m_{1}\right)\right) q_{1}^{2} \\
& +2\left(-\left(\left(-\frac{1}{2} m_{2} b+\frac{3}{2} q_{2}\right) r_{1}+a m_{2} d+\frac{1}{2} m_{1}\left(b m_{2}-3 q_{2}\right)\right) e c\right. \\
& \left.+a\left(\left(\left(-m_{2}+r_{2}\right) b+q_{2}\right) r_{1}+d\left(m_{2}-r_{2}\right) a+\left(\left(m_{2}-r_{2}\right) b-q_{2}\right) m_{1}\right)\right) c\left(r_{1}-m_{1}\right) q_{1} \\
& \left.\left.-q_{2} c^{2}\left(r_{1}-m_{1}\right)^{3}(-c e+a)\right) K_{2}-2 a^{2} r_{2}\left(r_{1}-m_{1}\right)\left(a d-\frac{3}{2} r_{1} b+\frac{3}{2} b m_{1}\right) q_{1}\right) K_{1}^{3} \\
& -2 a\left(\left(\left(-m_{2} e c+\frac{1}{2} a\left(m_{2}-r_{2}\right)\right) q_{1}-c\left(-m_{2} e c+a\left(m_{2}-r_{2}\right)\right)\left(r_{1}-m_{1}\right)\right) K_{2}+a^{2} r_{2}\left(r_{1}-m_{1}\right)\right) q_{1} r_{1} K_{1}^{2}, \\
& a_{0}=a\left(\left(q_{1}-c\left(r_{1}-m_{1}\right)\right)\left(m_{2} e q_{1}+\left(-m_{2} e c+a\left(m_{2}-r_{2}\right)\right)\left(r_{1}-m_{1}\right)\right) K_{2}+a^{2} r_{2}\left(r_{1}-t m_{1}\right)^{2}\right) q_{1} K_{1}^{3} \text {. }
\end{aligned}
$$

The polynomial $q(y)$ in Section 3.2 is $q(y)=\sum_{k=0}^{5} b_{k} y^{k}$, where coefficients are

$$
\begin{aligned}
b_{5}= & q_{2} c^{2}\left(K_{1} d+r_{1}\right) r_{2} K_{2}+K_{1} b^{2} q_{1} r_{2}^{2} \\
b_{4}= & q_{2} c^{2}\left(K_{1} d+r_{1}\right)\left(m_{2}-r_{2}\right) K_{2}^{2}+\left(\left(\left(c^{2} d e+2 a c d-2\left(\left(-\frac{1}{2} r_{1}+\frac{1}{2} m_{1}\right) c+q_{1}\right) b\right) K_{1}\right.\right. \\
& \left.\left.+2\left(\frac{1}{2} c e+a\right) c r_{1}\right) q_{2}+2 q_{1} b^{2} K_{1}\left(m_{2}-r_{2}\right)\right) r_{2} K_{2} \\
b_{3}= & \left(\left(\left(-r_{1}+m_{1}\right) c+q_{1}\right) K_{1} q_{2}^{2}+\left(\left(2\left(m_{2}-\frac{1}{2} r_{2}\right) c^{2} d e+2\left(m_{2}-r_{2}\right)\left(a c d-\left(\left(-\frac{1}{2} r_{1}\right.\right.\right.\right.\right.\right. \\
& \left.\left.\left.\left.\left.+\frac{1}{2} m_{1}\right) c+q_{1}\right) b\right)\right) K_{1}+2\left(c\left(m_{2}-\frac{1}{2} r_{2}\right) e+a\left(m_{2}-r_{2}\right)\right) c r_{1}\right) q_{2} \\
& \left.+q_{1} b^{2} K_{1}\left(m_{2}-r_{2}\right)^{2}\right) K_{2}^{2}+\left(\left(\left(\left(2 a c d-2\left(\left(-\frac{1}{2} r_{1}+\frac{1}{2} m_{1}\right) c+q_{1}\right) b\right) e\right.\right.\right. \\
& \left.\left.\left.+\left(a d+b\left(r_{1}-m_{1}\right)\right) a\right) K_{1}+a r_{1}(2 c e+a)\right) q_{2}+2 m_{2} q_{1} b^{2} e K_{1}\right) r_{2} K_{2} \\
b_{2}= & \left(-\left(\left(\left(2 r_{1}-2 m_{1}\right) c-2 q_{1}\right) e+a\left(r_{1}-m_{1}\right)\right) K_{1} q_{2}^{2}+\left(\left(m_{2} c^{2} d e^{2}+4\left(m_{2}-\frac{1}{2} r_{2}\right)\left(a c d-\left(\left(-\frac{1}{2} r_{1}\right.\right.\right.\right.\right.\right.
\end{aligned}
$$




$$
\begin{aligned}
& \left.\left.\left.\left.+\frac{1}{2} m_{1}\right) c+q_{1}\right) b\right) e+\left(m_{2}-r_{2}\right)\left(a d+b\left(r_{1}-m_{1}\right)\right) a\right) K_{1}+\left(m_{2} c^{2} e^{2}+4 a\left(m_{2}-\frac{1}{2} r_{2}\right) c e\right. \\
& \left.\left.\left.+a^{2}\left(m_{2}-r_{2}\right)\right) r_{1}\right) q_{2}+2 m_{2} q_{1} b^{2} e K_{1}\left(m_{2}-r_{2}\right)\right) K_{2}^{2}+a q_{2} e\left(\left(a d+b\left(r_{1}-m_{1}\right)\right) K_{1}+a r_{1}\right) r_{2} K_{2} \\
b_{1}= & 2 e\left(-\left(\left(\left(\frac{1}{2} r_{1}-\frac{1}{2} m_{1}\right) c-\frac{1}{2} q_{1}\right) e+a\left(r_{1}-m_{1}\right)\right) K_{1} q_{2}^{2}+\left(\left(m _ { 2 } \left(a c d-\left(\left(-\frac{1}{2} r_{1}\right.\right.\right.\right.\right.\right. \\
& \left.\left.\left.\left.\left.+\frac{1}{2} m_{1}\right) c+q_{1}\right) b\right) e+\left(a d+b\left(r_{1}-m_{1}\right)\right) a\left(m_{2}-\frac{1}{2} r_{2}\right)\right) K_{1}+a\left(m_{2} e c+a\left(m_{2}-\frac{1}{2} r_{2}\right)\right) r_{1}\right) q_{2} \\
& \left.+\frac{1}{2} m_{2}^{2} q_{1} b^{2} e K_{1}\right) K_{2}^{2} \\
b_{0}= & \left.-K_{1}\left(r_{1}-m_{1}\right) q_{2}+m_{2}\left(\left(a d+b\left(r_{1}-m_{1}\right)\right) K_{1}+a r_{1}\right)\right) a q_{2} e^{2} K_{2}^{2} .
\end{aligned}
$$

\section{Data Availability}

The data used to support the findings of this study are included within the article.

\section{Conflicts of Interest}

The authors declare that there are no conflicts of interest regarding the publication of this paper.

\section{Acknowledgments}

The authors thank teachers Min Zhao and Chuanjun Dai. This study was supported by the National Natural Science Foundation of China (grant nos. 31570364 and 61871293) and the National Key Research and Development Program of China (grant no. 2018YFE0103700).

\section{References}

[1] S. T. Wang, H. G. Yu, and C. J. Dai, “The dynamical behavior of a predator-prey system with holling type II functional response and allee effect," Applied Mathematics, vol. 11, no. 5, pp. 407-425, 2020.

[2] G. Wang, X. G. Liang, and F. Z. Wang, "The competitive dynamics of populations subject to an Allee effect," Ecological Modelling, vol. 124, no. 2-3, pp. 183-192, 1999.

[3] S. R. Zhou and G. Wang, "Allee-like effects in metapopulation dynamics," Mathematical Biosciences, vol. 189, no. 1, pp. 103-113, 2004.

[4] P. Aguirre, J. D. Flores, and E. González-Olivares, "Bifurcations and global dynamics in a predator-prey model with a strong Allee effect on the prey, and a ratio-dependent functional response," Nonlinear Analysis: Real World Applications, vol. 16, pp. 235-249, 2014.

[5] E. Gonzlez-Olivares and A. Rojas-Palma, "Limit cycles in a Gause-type predator-prey model with sigmoid functional response and weak Allee effect on prey," Mathematical Methods in Applied Sciences, vol. 35, pp. 963-975, 2012.

[6] J. Zu, "Global qualitative analysis of a predator-prey system with Allee effect on the prey species," Mathematics and Computers in Simulation, vol. 94, pp. 33-54, 2013.

[7] J. $\mathrm{Zu}$ and M. Mimura, "The impact of Allee effect on a predator-prey system with Holling type II functional response," Applied Mathematics and Computation, vol. 217, no. 7, pp. 3542-3556, 2010.

[8] Y. Cai, C. Zhao, W. Wang, and J. Wang, "Dynamics of a Leslie-Gower predator-prey model with additive Allee effect," Applied Mathematical Modelling, vol. 39, no. 7, pp. 20922106, 2015.

[9] D. L. DeAngelis, R. A. Goldstein, and R. V. O’Neill, “A model for tropic interaction,” Ecology, vol. 56, no. 4, pp. 881-892, 1975.

[10] J. R. Beddington, "Mutual interference between parasites or predators and its effect on searching efficiency," The Journal of Animal Ecology, vol. 44, no. 1, pp. 331-340, 1975.

[11] M. Fan and Y. Kuang, "Dynamics of a nonautonomous predator-prey system with the Beddington-DeAngelis functional response," Journal of Mathematical Analysis and Applications, vol. 295, no. 1, pp. 15-39, 2004.

[12] P. J. Pal and P. K. Mandal, "Bifurcation analysis of a modified Leslie-Gower predator-prey model with Beddington-DeAngelis functional response and strong Allee effect," Mathematics and Computers in Simulation, vol. 97, pp. 123-146, 2014.

[13] H. Mainul, "A detailed study of the Beddington-DeAngelis predator-prey model," Mathematical Biosciences, vol. 234, pp. 1-16, 2011.

[14] J. P. Tripathi, S. Abbas, and M. Thakur, "Dynamical analysis of a prey-predator model with Beddington-DeAngelis type function response incorporating a prey refuge," Nonlinear Dynamics, vol. 80, no. 1-2, pp. 177-196, 2015.

[15] J. Lee and H. Baek, "Dynamics of a Beddington-DeAngelis type predator-prey system with constant rate harvesting," Electronic Journal of Qualitative Theory of Differential Equations, vol. 1, no. 1, pp. 1-20, 2017.

[16] G. Birkhoff and G. C. Rota, Ordinary Differential Equations Introductions to Higher Mathematics, Ginn and Company, Boston, MA, USA, 1962.

[17] F. D. Chen, "On a nonlinear nonautonomous predator-prey model with diffusion and distributed delay," Journal of Computational and Applied Mathematics, vol. 180, no. 1, pp. 33-49, 2005.

[18] Z. F. Zhang, T. R. Ding, W. Z. Huang, and Z. X. Dong, Qualitative Theory Of Differential Equations (Translations Of Mathematical Monographs), Vol. 101, American Mathematical Society, Providence, RI, USA, 1992.

[19] D. R. Merkin, F. F. Afagh, and A. L. Smirnov, Introduction to the Theory of Stability, Springer, Berlin, Germany, 1997.

[20] C. Chicone, Ordinary Differential Equations with Applications, Springer, Berlin, Germany, 2006. 
[21] A. A. Andronov, E. A. Leontovich, I. I. Gordon, and A. G. Maier, Qualitative Theory of Second-Order Dynamic Systems, Halsted Press, New York, NY, USA, 1973.

[22] L. Perko, Differential Equations and Dynamical Systems, Springer, Berlin, Germany, 2001.

[23] B. Pirayesh, A. Pazirandeh, and M. Akbari, "Local bifurcation analysis in nuclear reactor dynamics by Sotomayor's theorem," Annals of Nuclear Energy, vol. 94, pp. 716-731, 2016.

[24] Y. A. Kuznetsov, Elements of Applied Bifurcation Theory, Springer, Berlin, Germany, 1998.

[25] Y. A. Kuznetsov, "Numerical normalization techniques for all codim 2 bifurcations of equilibria in ODE's," SIAM Journal on Numerical Analysis, vol. 36, no. 4, pp. 1104-1124, 1999.

[26] Q. X. Ye, Z. Y. Li, M. X. Wang, and Y. P. Wu, Introduction to Reation-Diffusion Equations, Science Press, Beijing, China, 2011.

[27] M. X. Wang and P. Y. H. Pang, "Qualitative analysis of a diffusive variable-territory prey-predator model," Discrete \& Continuous Dynamical Systems-A, vol. 23, no. 3, pp. 10611072, 2009.

[28] M. Pascual, "Diffusion-induced chaos in a spatial predatorprey system," Proceedings of the Royal Society of London B, vol. 251, pp. 1-7, 1993.

[29] F. Q. Yi, J. J. Wei, and J. P. Shi, "Bifurcation and spatiotemporal patterns in a homogeneous diffusive predator-prey system," Journal of Differential Equations, vol. 246, no. 5, pp. 1944-1977, 2009.

[30] A. Y. Wan, Z. Q. Song, and L. F. Zheng, "Patterned solutions of a homogenous diffusive predator-prey system of holling type-III," Acta Mathematicae Applicatae Sinica (English Scries), vol. 32, no. 1, pp. 1073-1086, 2016. 